\title{
Teamwork and Individual Productivity
}

\author{
Flores-Szwagrzak, Karol ; Treibich, Rafael
}

Document Version

Accepted author manuscript

Published in:

Management Science

DOI:

10.1287/mnsc.2019.3305

Publication date:

2020

License

Unspecified

Citation for published version (APA):

Flores-Szwagrzak, K., \& Treibich, R. (2020). Teamwork and Individual Productivity. Management Science, 66(6), 2523-2544. https://doi.org/10.1287/mnsc.2019.3305

Link to publication in CBS Research Portal

\section{General rights}

Copyright and moral rights for the publications made accessible in the public portal are retained by the authors and/or other copyright owners and it is a condition of accessing publications that users recognise and abide by the legal requirements associated with these rights.

Take down policy

If you believe that this document breaches copyright please contact us (research.lib@cbs.dk) providing details, and we will remove access to the work immediately and investigate your claim. 


\title{
Teamwork and Individual Productivity
}

\author{
Karol Flores-Szwagrzak* Rafael Treibich**
}

\begin{abstract}
We propose a new method to disentangle individual from team productivity, CoScore. CoScore uses the varying membership and levels of success of all teams to infer, simultaneously, an individual's productivity and her credit for each of her teams' successes. Crucially, the productivities of all individuals are determined endogenously via the solution of a fixed point problem. We show that CoScore is well defined and provide axiomatic foundations for the inferred credit allocation. We illustrate CoScore in scientific research and sports.
\end{abstract}

Keywords: Networks, Collaboration, Productivity indices, Ranking methods

\section{INTRODUCTION}

In January of 2017 Futbol Club Barcelona fired Pere Gratacós for stating that Lionel Messi, the team's superstar, "would not be as good" if not for his superlative teammates. Despite leading Barcelona to several national and continental championships, Messi has always struggled with Argentina's national team. One might then wonder if it is really Messi who explains Barcelona's success or rather, as Gratacós' statement suggests, Barcelona that explains Messi's success. Even when coming from well informed experts, judgements of this type are inherently subjective. The collective nature of the game means that a player's productivity cannot be reduced to either her individual statistics or her winning record. It should capture the player's contribution to the success of her team, while accounting for the quality of her teammates. Ideally, this would require observing the counterfactual success of the team absent a particular player, which is impossible. How can we then systematically

Date: April 11, 2019.

*Copenhagen Business School, ksz.eco@cbs.dk

**University of Southern Denmark, rtr@sam.sdu.dk

We thank Carlos Alós-Ferrer, Sophie Bade, Miguel Ballester, Albert-László Barabási, Salvador Barberà, Yann Bramoullé, Manel Baucells, Christopher P. Chambers, Gabrielle Demange, Enrico Diecidue, Jens Gudmundsson, Lene Holbæk, Özgür Kıbrıs, Livia Helen, Jean-François Laslier, Isabelle Mairey, Michael Mandler, François Maniquet, Alan D. Miller, Juan D. Moreno-Ternero, Hervé Moulin, Klaus Ritzberger, Tomás Rodríguez Barraquer, Alexander Schandlbauer, William Thomson, Armando Treibich, Rodrigo Velez, Oscar Volij, and Lars Peter Østerdal for helpful comments. We also thank seminar audiences in Barcelona, Bordeaux, Cologne, Copenhagen, London, Louvain-la-Neuve, Lund, Maastricht, Odense, Rochester and Seoul. 
evaluate the productivity of a player on the basis of her team's success, that is, on the basis of work not done entirely by herself?

The question is obviously relevant beyond sports. Many production processes require teamwork, yet individual contributions are not perfectly, if at all, observable; this has been the main impetus behind the study of "moral hazard" in teams (Holmstrom, 1982; McAfee and McMillan, 1991; Guillen et al., 2014). Even formerly individual areas of production, such as science and innovation, are increasingly dominated by teams; the vast majority of publications are now coauthored and the average number of coauthors is steadily increasing (Wuchty et al., 2007; Jones, 2009); teams also achieve higher impact research (Wuchty et al., 2007), are more likely to avoid failures, and achieve innovation breakthroughs (Singh and Fleming, 2010). Not surprisingly, since the individual contributions of researchers cannot be observed, the attribution of credit for collaborative scientific research is neither well understood nor explicit (Gans and Murray, 2013, 2014). Researchers are nonetheless evaluated on the basis of their increasingly collaborative research for professional advancement, compensation, and recognition (Sauer, 1988; Bikard et al., 2015).

In this paper, we evaluate individual productivity in a simple theoretical model where team membership and team production are observable but individual contributions towards team production are not. The main idea is twofold: Firstly, quantifying individual productivity requires knowing how much the individual contributes to her teams. Secondly, determining how much she contributes itself requires knowing her individual productivity since the more productive individuals bring more expertise, team-building skills, and visibility, and contribute more on average. This could mean that quantifying individual productivity is impossible in the absence of information on individual contributions; conversely, it could mean that quantifying individual contributions is impossible in the absence of information on individual productivity. To the contrary, we show that both problems can be thought of as dual and solved jointly.

We propose a new method, CoScore, building on this idea, by jointly scoring or co-scoring individual performance and credit for team productivity. Individual credit on a given project is understood as the fraction of the team output attributed to the individual for that project. CoScore defines an individual's productivity score as the sum of her credits over all the projects she has contributed to. In turn, on each project, credit is allocated proportionally to the score of each teammate. Crucially, the scores and the credits of all individuals are determined endogenously and simultaneously as the solution of a fixed point problem.

Formally, our model consists of a database of projects $C$ undertaken by teams composed of individuals drawn from $N$. Each project $p \in C$ is described by the team of individuals $T(p) \subseteq N$ undertaking the project and a numerical measure of output or worth $w(p) \in \mathbb{R}_{+}$. 
Importantly, we take this measure as given to focus on the assessment of individual credit and productivity. Each individual, $i \in N$, can thus be described by her production record, $C_{i} \subseteq C$, specifying the set of projects she participated in. CoScore defines the productivity score of each individual by the system of equations

$$
s_{i}=\sum_{p \in C_{i}} \frac{s_{i}}{\sum_{j \in T(p)} s_{j}} w(p) \quad \forall i \in N
$$

where $s_{i}$ is the score of individual $i$. The score of each individual thus depends on the (endogenous) scores of her teammates. CoScore is well defined whenever each individual in the database has at least one solo project with strictly positive worth, no matter how small: for each $i \in N$, there is $p \in C$ such that $T(p)=\{i\}$ and $w(p)>0$. This is a sufficient condition for the system of equations (1) to have a unique solution. ${ }^{1}$ Existence follows from Brouwer's fixed point theorem; uniqueness comes from the fact that a solution of (1) maximizes a strictly concave function over an open and convex set (see Theorem 3 in Appendix A). CoScore can be extended to tackle applications like team sports where solo projects are not possible or where not every individual has such a project (see Section 3).

CoScore reflects the structure of the entire team membership network and the records of possibly all teams in the database. ${ }^{2}$ The central insight is that allocating credit on a given project requires assessing the productivity of each member in the team. Thus, the credit assigned to an individual depends on the credit assigned to all of her teammates on all of their projects, which is itself determined by their teammates' credit on all of their respective projects, and so forth. Hence, an individual's credit on a project depends on the whole database, not simply on how many teammates she has. This is in sharp contrast to more naive productivity measures such as the egalitarian score, where credit is allocated equally among the individuals in each team,

$$
s_{i}^{e}=\sum_{p \in C_{i}} \frac{1}{\# T(p)} w(p) \quad \forall i \in N
$$

Measures such as the egalitarian score can only control for collaboration anonymously, based solely on the number of teammates in a project, not on their characteristics or any other information in the database.

\footnotetext{
${ }^{1}$ Technical assumptions of this type are common to fixed point based methods used for internet search engines, social networks, general equilibrium, game theory, etc.

${ }^{2}$ Here, we take the team membership network to be the weighted hyper-graph where nodes represent different players and hyper-edges represent projects of varying weights.
} 
Axiomatic analysis. The CoScore and the egalitarian score take different stances on how to allocate credit for teamwork, resulting in different measures of individual productivity. There are, of course, many other conceivable ways to allocate credit. We thus study the basic problem from a general perspective, by evaluating all the possible credit allocation rules using the axiomatic approach. Previously, the axiomatic approach has bee used extensively to motivate otherwise ad hoc resource allocation rules (Young, 1994; Thomson, 2001). Our axioms formalize basic properties that a credit allocation rule should obey and describe their joint implications, singling out specific credit allocation rules.

In Section 2, we present axiomatic characterizations of the credit allocation rules associated to the CoScore and the egalitarian score. The characterizations highlight the fundamental differences between these rules: The egalitarian rule is the only anonymous rule ensuring that an indivdiual's credit depends solely on her own record, not on any other information in the database; hence, it is the only anonymous rule that can be computed for each individual in isolation, accounting only for the number of individuals in each project (Theorem 1). In contrast, the axiom satisfied by the CoScore rule but not by the egalitarian rule, "aggregate strength," captures the idea that the inferred credit of an individual on a project should depend on how strong she is relative to her teammates. It requires an individual's credit in a given project to depend on the aggregate record or "strength" of her teammates, but not necessarily on their number. We prove that the CoScore rule is characterized by aggregate strength and two other axioms also satisfied by the egalitarian rule (Theorem 2).

Extensions and empirical case studies. Our axiomatic analysis tackles a stylized model where only team memberships and outputs are observable and each individual has a solo project of strictly positive worth. In specific applications, teamwork involves further structure and observable information: the roles of teammates, their degree of participation, differences in the time horizon of their production records, etc. Moreover, individuals may not have solo projects as in team sports. CoScore can be extended to account for these additional features.

To illustrate, in Section 3, we carry out two empirical case studies in scientific research and team sports. For research, we analyse a database consisting of all articles from 33 major economics journals over the period 1970 - 2015; for sports, we analyse a database consisting of the yearly records of all NBA teams over the period $1946-2011$.

In both cases, individuals (researchers or players) have different observable ages, some are at the beginning of their careers while others have reached maturity. Mechanically, the production record of senior individuals tends to be lengthier since they have had more time to accumulate projects. To account for age, we can define the score of each individual as her 
average credit over the span of her career, instead of her total credit. This is more reflective of individual productive ability, allowing for meaningful comparisons across individuals of different career lengths.

In the NBA case, we also have data on the degree of participation in a team, the amount of time each player spent on the court. It is natural to extend CoScore to incorporate this information. We propose a family of scores parametrized by a scalar $\alpha \in[0,1]$, where a fraction $\alpha$ of credit in each project is allocated proportionally to individual time participation and the remaining fraction $(1-\alpha)$ is allocated proportionally to her endogenous productivity weighted by her time participation. If $\alpha$ takes a value of one, all credit is allocated proportionally to the degree of participation and we arrive at an egalitarian-like score correcting for differences in time participations: if all time participations are equal, credit is shared equally. At the other extreme, if $\alpha$ takes a value of zero, all credit is allocated proportionally to the endogenous productivity weighted by time participation and we obtain a score correcting for differences in the degree of participations that is closer to CoScore. The parameter $\alpha$ thus quantifies the extent to which the allocation of credit depends on the endogenous productivity. In the scientific research case, we assume project participation to be binary since we can only observe project membership but not time participation; here a share $\alpha$ of each project is allocated equally among its members while the remaining $(1-\alpha)$ share is allocated proportionally to their endogenous productivity.

To determine the significance of the choice of $\alpha$, we perform a sensitivity analysis assessing how the parameter affects the productivity scores and the corresponding rankings of individuals. In both applications, we observe a sharp divide between the parametric extensions of CoScore and the egalitarian benchmark. Thus, accounting for the production records of all team members in the attribution of credit results in markedly different scores and rankings.

Although CoScore and its extensions cannot be computed independently for each individual, since they require looking at the whole database of projects, their implementation is straightforward. Like Google's PageRank algorithm (Page et al., 1998), they can be applied to extremely large databases in a systematic way, providing a concrete alternative to measure individual productivity.

Related work. The endogenous formulation of CoScore as a fixed point and its intensive use of the team membership network are reminiscent of various measures of network centrality used to rigorously quantify socioeconomic phenomena such as reputation, importance, popularity, and intelectual influence. ${ }^{3}$ For example, "eigenvector centrality" (Bonacich, 1972) defines the "centrality" of an individual in a social network to be proportional to the sum

\footnotetext{
${ }^{3}$ For a survey on network centrality measures see Jackson (2008) and Newman (2010).
} 
of the centralities of her neighbors. Such measures have proven useful in many different contexts, as shown in particular with the PageRank algorithm used by Google to rank the relevance of web pages (Page et al., 1998) or the recursive methods to rank journals (Pinski and Narin, 1976; Palacios-Huerta and Volij, 2004; Demange, 2014).

Rigorous rankings of sports teams also have an endogenous nature since a team is deemed to be "successful" not only on the basis of its wins or scores, but perhaps most importantly, if it can defeat other "successful" teams. Like eigenvector centrality for social networks, these rankings rely on the Perron-Frobenius theorem and fixed point methods (Keener, 1993).

CoScore relies on similar insights but differs substantially from network centrality because it is not defined on a network but on a weighted hypergraph, the database of projects. Furthermore, while the centrality of a given player increases with the centrality of her neighbors, the credit of a given player (according to CoScore) decreases with the productivity of her teammates. ${ }^{4}$

The application of CoScore to scientific research also contributes to a growing literature on the measurement of individual academic productivity and intellectual influence. The remarkable uptake of the $h$-index (Hirsch, 2005), now routinely reported by Google Scholar, attests to the demand for simple indices summarizing a researcher's publication or citation record. It has also motivated a number of alternatives (Egghe, 2006; Lehmann et al., 2006), some of which have been supported axiomatically (Chambers and Miller, 2014; Perry and Reny, 2016). CoScore differs from all these measures in that it does not simply summarize an individual record; it summarizes all records simultaneously. CoScore thus reflects the collaborative nature of scientific production, now dominated by coauthorship (Wuchty et al., 2007). In contrast, indices summarizing an individual's record alone must either ignore the fact that papers are coauthored or account for it using only paper features such as the number of coauthors (Marchant, 2009). ${ }^{5}$

Potential applications. CoScore and the measures derived from it can also be applied to situations where teamwork is understood more generally. For instance, it is well known that product bundling increases revenue compared to selling the bundle's components separately (Adams and Yellen, 1976; McAfee et al., 1989). A bundle can then be seen as a form of

\footnotetext{
${ }^{4}$ Note, from a more technical point of view, that CoScore is not defined by a system of linear equations and does not rely on the Perron-Frobenius theorem.

${ }^{5}$ The biases of these approaches are well known. Assigning full credit to all coauthors, artificially inflates their publication record by overrating the value of coauthored papers (Price, 1981; Liebowitz, 2013). This favors researchers with multiple collaborators or belonging to large teams and gives perverse incentives for artificial coauthorship. Dividing credit uniformly corrects this bias but dilutes the credit due to the intellectual leaders of a paper, ignoring that authors' contributions may differ substantially (Hagen, 2008; Shen and Barabási, 2014). The same concern applies to the various proposals to allocate credit when authors are listed by order of importance or by other field-specific conventions (Hagen, 2008; Stallings et al., 2013).
} 
teamwork between its components; its price is observable and takes the role of our measure of worth and we can asses the share of the price due to each component by observing variations in the prices of bundles including the component. From a managerial perspective, this can inform component purchase decisions and the design of new bundles. Similarly, digital platforms such as Hulu, iTunes, Netflix, and Spotify charge users different access fees for bundles of entertainment content; here, the platform manager can assess the relative benefits and costs of including or removing content.

Museum passes or city public transportation cards also offer buyers access to bundles of goods. Each component contributes differently to the value of the pass or card to consumers but this is not immediately observable. Assessing the differing contributions is nonetheless essential to fairly compensate the providers of the various components (Ginsburgh and Zang, 2003; Bergantiños and Moreno-Ternero, 2015).

Finally, a closely related problem is that of sharing sports event broadcasting revenues among participating teams (Bergantiños and Moreno-Ternero, 2018). Here the individuals engaging in teamwork are the sports teams and the value they produce is the broadcasting revenue of their joint games. Analytically, the key difficulty is that teams may contribute differently to produce the joint revenue but this cannot be observed.

\section{Axiomatic Analysis}

We now study credit allocation from an axiomatic perspective. We provide axiomatic characterizations of the credit allocation rules associated to the egalitarian score and CoScore. The axioms we introduce describe how the allocation of credit is affected by changes in team composition and output in the database of projects. We thus start by introducing a model allowing us to vary these parameters and thereby relate credit allocation across the corresponding databases.

The axiomatic analysis maintains the assumption that each individual has at least one solo project with strictly positive worth, the same condition that ensures CoScore is welldefined. This assumption is not satisfied in some applications such as team sports. However, the analysis here illuminates the main properties underlying CoScore and its extensions to general real-life applications (Section 3), contrasting these with the egalitarian benchmark. ${ }^{6}$

\footnotetext{
${ }^{6}$ Similarly, the axiomatic analysis of PageRank (Altman and Tennenholtz, 2005) concerns its simplest version which is not always well defined; a technical condition is also imposed to ensure it is (the directed hyperlink graph must be strongly connected). The axiomatic analysis of the "invariant method" (PalaciosHuerta and Volij, 2004) closely related to PageRank is also carried out under the analogous assumption that one can get from one journal to any other journal by a directed chain of citations; this yields the "irreducible matrix" condition necessary for the well-definedness of the invariant method.
} 
2.1. Model. A database $D$ consists of a finite collection of projects $C(D)$. Each project $p \in C(D)$ is described by the team of individuals $T(p, D)$ involved in it and a measure of output or worth $w(p, D) \in \mathbb{R}_{+}$. We refer to $p \in C(D)$ as a collaborative project when team $T(p, D)$ contains more than one individual. The collection of all individuals involved in projects in database $D$ is denoted by $N(D)$. The production record of individual $i \in N(D)$ is denoted by $C_{i}(D)$ and consists of all projects involving her, all $p$ such that $i \in T(p, D)$. Similarly, the production record of team $S \subseteq N(D)$ is denoted by $C(S, D)$ and consists of all projects involving team $S$, all $p$ such that $S=T(p, D)$. Throughout this section we assume that each individual has at least one solo project with strictly positive yet possibly arbitrarily small worth.

A credit allocation for project $p$ in database $D$ is a profile $z(p) \in \mathbb{R}_{+}^{N(D)}$ distributing the project's output among its team members, $\sum_{i \in T(p, D)} z_{i}(p)=w(p, D)$. We thus exclude the possibility that, for example, two individuals each receive more than half of the credit for a project. $^{7}$ This is, however, without loss of generality and our results can be modified to reflect more general assumptions whereby the total credit on a collaborative project depends on the team's size and output. ${ }^{8}$ We denote by $Z(p, D)$ all the possible credit allocations for project $p$ in database $D$. A credit allocation for database $D$ specifies a credit allocation for each project in the database; we denote by $Z(D)$ the possible credit allocations for database $D, Z(D) \equiv \times_{p \in C(D)} Z(p, D)$.

A database involving three individuals and a corresponding credit allocation are illustrated in Figure 1. Projects are represented by rectangles of varying sizes proportional to their worth. A credit allocation specifies the share of credit on each project due to each team member; the credit of a member can thus be represented as the area within a rectangle assigned to her. A particular credit allocation can then be illustrated as a "colouring" of each of the rectangles as depicted in Figure 1 (ii). Note that the share of credit allocated to each individual need not a priori be the same across projects, even if these involve the same team; for instance, in Figure 1 (ii), B gets a larger share of credit in her larger joint project with A.

2.2. Rules. A (credit allocation) rule recommends a credit allocation for each database. Formally, a rule $r$ is a function mapping each database $D$ into a credit allocation $r(D) \in$

\footnotetext{
${ }^{7}$ Instead of being part of the definition of a credit allocation, this assumption could instead be explicitly stated as an axiom and the ensuing analysis would be unchanged.

${ }^{8}$ For example, Bikard et al. (2015) argue that the implicit attribution of credit in scientific research is consistent with valuing an $n$-member project at $\sqrt{n}$ times its output. In scientific research, a project is a paper, the team undertaking it is constituted by its coauthors, and its output or worth is understood as its number of citations.
} 


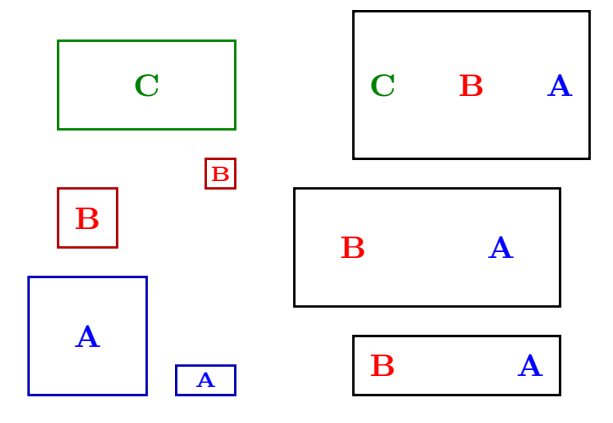

(i) Database: Projects are represented by rectangles of varying sizes proportional to their worth. Ann has two solo projects of worth 16 and 2, Bob has two solo projects of worths 4 and 1, Cat has one solo project of worth 18. Ann and Bob have two joint projects of worths 36 and 14. Finally, Ann, Bob and Cat have a joint project of worth 45 .

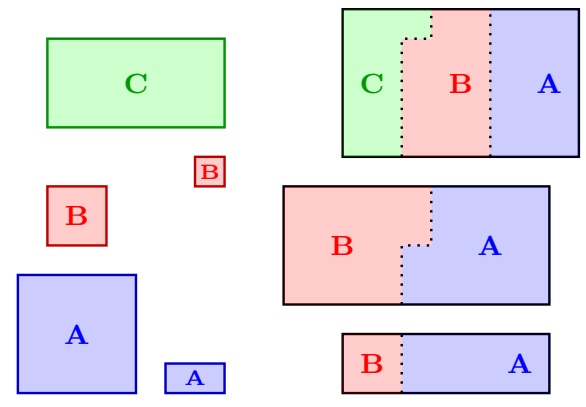

(ii) Credit allocation: The credit allocated to each individual is represented by the corresponding shaded area. The credit for each of the solo projects goes entirely to the corresponding individual. The total credit allocated on a joint project equals the project's worth.

Figure 1. Database and credit allocation rule.

$Z(D)$. We denote by $r_{i}(p, D)$ the credit assigned by rule $r$ to individual $i$ for project $p$ in database $D$ and by $r(p, D)$ the profile $\left(r_{i}(p, D)\right)_{i \in N(D)}$.

The most basic rule, the egalitarian rule, allocates the worth of each project among its team members equally, irrespective of all other information available in the database: for each database $D$, each $p \in C(D)$, and each $i \in T(p, D)$,

$$
r_{i}(p, D)=\frac{1}{\# T(p, D)} w(p, D)
$$

The CoScore rule allocates credit on each project proportionally to the productivity score of each member of the team, as measured endogenously by the CoScore in the relevant database: for each database $D$, each $p \in C(D)$, and each $i \in T(p, D)$,

$$
r_{i}(p, D)=\frac{s_{i}(D)}{\sum_{j \in T(p, D)} s_{j}(D)} w(p, D)
$$

where $s_{i}(D)$ denotes the score of individual $i$ in database $D$ as measured by CoScore,

$$
s_{i}(D)=\sum_{p \in C_{i}(D)} \frac{s_{i}(D)}{\sum_{j \in T(p, D)} s_{j}(D)} w(p, D) \quad \forall i \in N(D)
$$

The CoScore rule is well defined because CoScore is (see Theorem 3 in Appendix A).

Remark 1. The CoScore rule can also be defined endogenously: For each database $D$ and each individual $i \in N(D)$, replace $s_{i}(D)$ with $\sum_{p \in C_{i}(D)} r_{i}(p, D)$ in the definition of the CoScore rule in equation (2). 
When there are only two individuals in the database, the CoScore rule allocates credit on joint projects proportionally to each individual's aggregate solo contribution. When the database contains more than two individuals, the CoScore rule does not generally admit a simple closed form representation. A notable exception is the case where each joint project in the database involves all individuals; there, the credit on joint projects is also allocated proportionally to each individual's aggregate solo contribution. Figure 2 illustrates both the egalitarian and the CoScore rules in the two-individual case.
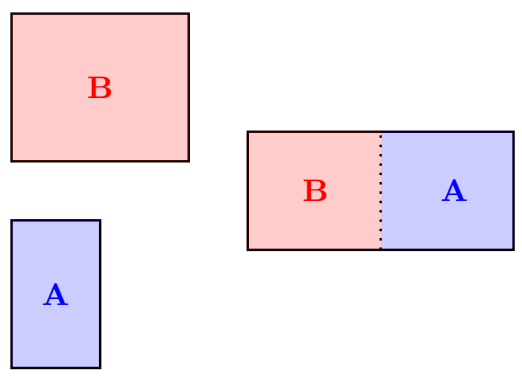

(i) Allocation of credit according to the egalitarian rule. $\mathrm{A}$ and $\mathrm{B}$ receive equal credit for their joint project.

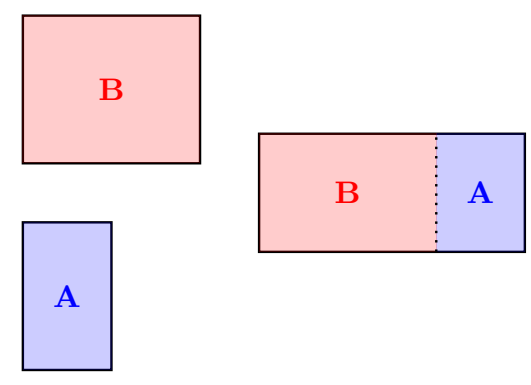

(ii) Allocation of credit according to the CoScore rule. The ratio between the credit received by $\mathrm{A}$ and $\mathrm{B}$ on their joint project is the same as the ratio between the worth of A's solo project and B's solo project.

Figure 2. Egalitarian and CoScore rules for a database with 2 individuals.

2.3. Axioms and characterizations. We first introduce the two axioms characterizing the egalitarian rule. The first axiom, "anonymity," requires credit to be allocated on the basis of information observable in the database only; exogenous characteristics associated to each individual's "name tag" should bear no influence. Thus, if we could switch the role of individuals $i$ and $j$ in a database by permuting their production records, then the credit assigned to $i$ on each project after the permutation should be the same as the credit which was assigned to $j$ before it, and conversely.

Anonymity: For each pair $D, D^{\prime}$, if $C(D)=C\left(D^{\prime}\right), w(p, D)=w\left(p, D^{\prime}\right)$ for each $p \in C(D)$, and $\pi: N(D) \rightarrow N\left(D^{\prime}\right)$ is a bijection such that $T\left(p, D^{\prime}\right)=\pi(T(p, D))$ for each $p \in C(D)$, then $r_{i}(p, D)=r_{\pi(i)}\left(p, D^{\prime}\right)$ for each $i \in N(D)$ and each $p \in C(D)$.

Anonymity is a basic property excluding arbitrary credit allocation rules. For example, it precludes a predetermined individual from receiving all credit on each of her collaborative projects. The egalitarian and CoScore rules are anonymous.

The second axiom, "own record only," requires that an individual's credit on a project should depend only on her own production record. Her credit on a project may however depend on the composition of her team, not just on its size. To state the axiom formally, we 
specify what it means for two collections of projects to be the same across two databases. For each pair $D, D^{\prime}$, each $C \subseteq C(D)$, and each $C^{\prime} \subseteq C\left(D^{\prime}\right)$, we say that $C$ and $C^{\prime}$ coincide if $C=C^{\prime}$ and, for each $p \in C, w(p, D)=w\left(p, D^{\prime}\right)$ and $T(p, D)=T\left(p, D^{\prime}\right)$.

Own record only: For each pair $D, D^{\prime}$ and each $i \in N(D) \cap N\left(D^{\prime}\right)$, if $C_{i}(D)$ and $C_{i}\left(D^{\prime}\right)$ coincide, then, for each $p \in C_{i}(D), r_{i}(p, D)=r_{i}\left(p, D^{\prime}\right)$.

The axiom requires that, if an individual has the same production record in two databases, then her credit on each of her projects in both databases is the same. Thus, a rule satisfies own record only if the allocation of credit to an individual remains unaffected by changes in the database that do not alter her production record. Clearly, the CoScore rule does not satisfy this axiom because the score of each individual depends possibly on all of the information in the database.

We can now present the characterization of the egalitarian rule.

Theorem 1. A rule satisfies anonymity and own record only if and only if it is the egalitarian rule.

The properties in Theorem 1 are logically independent. See Appendix D.

We now introduce the three axioms characterizing the CoScore rule. The first axiom specifies that a rule's credit allocation behavior is "consistent" in the following sense: Upon removing an individual from the database, keeping fixed the characteristics of each project not involving her, the allocation of credit among the remaining individuals is unaffected. Removing an individual obviously requires removing her individual projects from the database. However, removing her collaborative projects as well would artificially reduce her teammates' production records. On the other hand, leaving her collaborative projects in the database, absent her participation, would artificially inflate her teammates' contribution. Thus, all of her collaborative projects should stay in the database, but their worth should be updated by subtracting the credit that was originally assigned to her.

To state the axiom formally, we now define the "reduced database" obtained after removing an individual from a database given a credit allocation. For each database $D$, each credit allocation $x \in Z(D)$ and each individual $k \in N(D)$, we denote by $D_{x}^{-k}$ the database obtained from $D$ by removing all of $k$ 's non-collaborative projects and, for each of $k$ 's collaborative projects, by withdrawing her from its team and reducing its worth by the credit assigned to her under $x$. Thus, $C\left(D_{x}^{-k}\right)=C(D) \backslash C(\{k\}, D)$ and, for each collaborative $p \in C_{k}(D)$,

$$
T\left(p, D_{x}^{-k}\right)=T(p, D) \backslash\{k\} \text { and } w\left(p, D_{x}^{-k}\right)=w(p, D)-x_{k}(p) .
$$


The team and worth of each project not involving $k$ are identical in $D_{x}^{-k}$ and $D$.

Consistency: For each $k \in N(D)$, each $i \in N(D) \backslash\{k\}$, and each $p \in C\left(D_{r(D)}^{-k}\right)$,

$$
r_{i}(p, D)=r_{i}\left(p, D_{r(D)}^{-k}\right) .
$$

Thus, rule $r$ is consistent if the credit allocation for all individuals other than $k$ is the same in the reduced database $D_{r(D)}^{-k}$ as in the original database $D$. Palacios-Huerta and Volij (2004) use a similar consistency property in their axiomatic characterization of the "invariant method" to rank academic journals. Their "reduced" journal database is obtained by reassigning the citations from the withdrawn journal in a predetermined way. In contrast, we readjust the worth of each project endogenously, since the reduction depends on the credit allocated to the withdrawn individual by the rule itself. ${ }^{9}$ Consistency properties have been used extensively in resource allocation and game theory (see Thomson, 2011, 2016, for surveys). The egalitarian and the CoScore rules are consistent. Consistency is illustrated in Figure 3 for a database with three individuals.

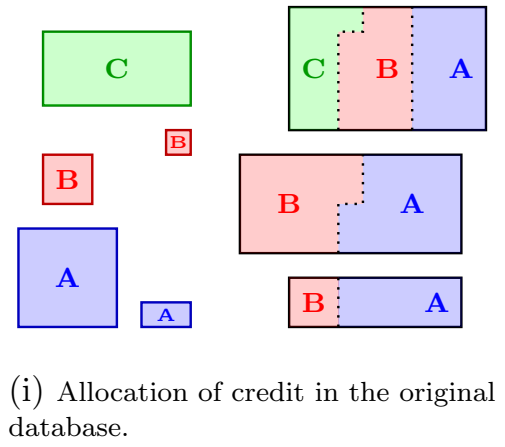

(i) Allocation of credit in the original database.

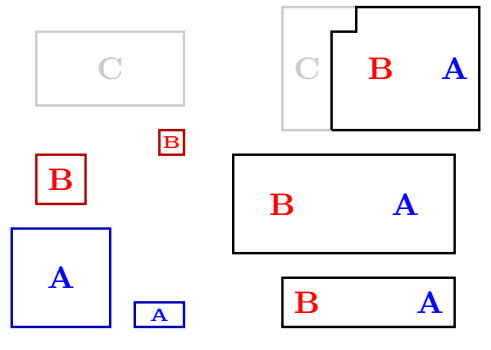

(ii) Reduced database after Cat is removed.

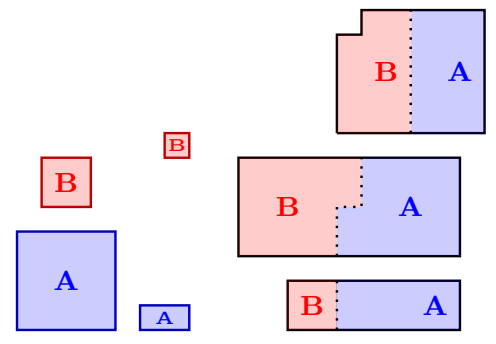

(iii) Consistency: Ann and Bob's credit on each of their projects remains unaffected.

Figure 3. Consistency

The second axiom, "aggregate worth," captures the idea that the credit allocation should only depend on the aggregate value produced by each team, not on this value's breakdown into projects of different worth. It requires that, for example, replacing two projects of worth 100 and 200 by a single project of worth 300, both involving the same team, does not affect the credit allocation.

Before stating the axiom formally, we define the database obtained after replacing a set of projects involving a team by a single project of equal aggregate worth involving the same team. For each database $D$, each team $S \subseteq N(D)$, and each set of projects involving that team $C \subseteq C(S, D)$, we denote by $D_{C}$ the database obtained by replacing all projects in $C$

\footnotetext{
${ }^{9}$ See Dequiedt and Zenou (2017) for an application of a consistency property in the characterization of a class of network centrality measures.
} 
by a single project $p_{C}$ involving team $S$ and with total worth equal to that of the sum of all projects in $C$, all else equal. ${ }^{10}$

Aggregate worth: For each $C \subseteq C(D)$ such that all projects in $C$ involve the same team, $r\left(p_{C}, D_{C}\right)=\sum_{q \in C} r(q, D)$ and, for each $p \in C\left(D_{C}\right) \backslash\left\{p_{C}\right\}, r\left(p, D_{C}\right)=r(p, D)$.

Thus, a rule satisfies aggregate worth if the credit allocated to each team member for the aggregate project $p_{C}$ in the new database $D_{C}$ is equal to the total credit that was previously allocated to her for all projects in $C$, and the allocation of credit on all other projects is the same as before. Aggregate worth is satisfied by the egalitarian and the CoScore rules. Aggregate worth is illustrated in Figure 4 for a database with three individuals.

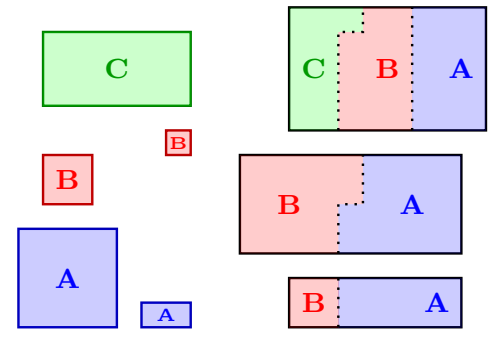

(i) Allocation of credit in the original database.

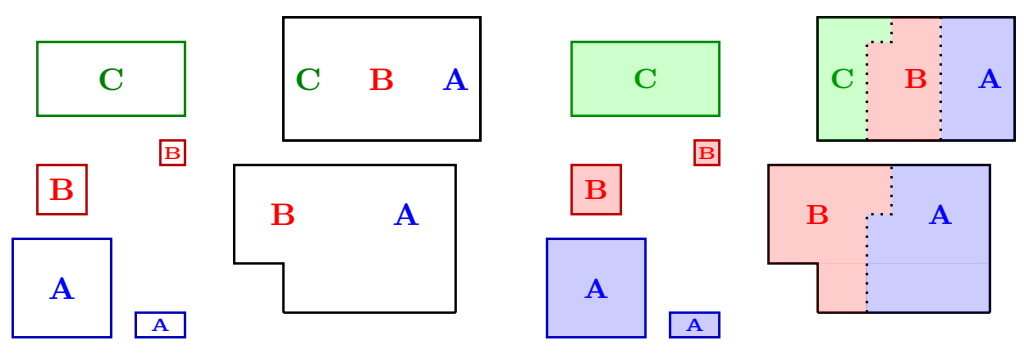

(ii) Reduced database after Ann and Bob's two joint projects are aggregated into a single project of equal total worth.

(iii) Aggregate Worth: Ann and Bob's credit on the aggregate project are equal to the total credit they were allocated on each of their two joint projects. The allocation of credit on all other projects remains unaffected.

Figure 4. Aggregate Worth

The last axiom, "aggregate strength," captures the idea that an individual's credit on a project should depend on how strong she is relative to her teammates, not just on how many teammates she has. More precisely, it requires the individual's credit to be unaffected if a group of her teammates on the project are replaced by a single "aggregate" teammate whose record comprises all the projects that previously involved members of that group. The individual's credit should depend only on her own record and the aggregate record of her teammates in that project, since their aggregate record reflects their overall strength.

The axiom only aggregates groups of teammates sharing the same collaborative projects, as it would be questionable to require the allocation of credit to remain unaffected if the collaborative projects involving the group varied considerably. Assume for instance that individual $C$ has two projects of identical worth, one with individual $A$ and one with individual

\footnotetext{
${ }^{10}$ Formally, for each $S \subseteq N(D)$ and each $C \subseteq C(S, D)$, database $D_{C}$ comprises the collection of projects $C\left(D_{C}\right)=[C(D) \backslash C] \cup\left\{p_{C}\right\}$, where project $p_{C}$ involves each member of $S, T\left(p_{C}, D_{C}\right)=S$, and has a worth equal to the total worth of the projects in $C, w\left(p_{C}, D_{C}\right)=\sum_{p \in C} w(p, D)$. For each project in $C(D) \backslash C$, its team and worth are identical in $D$ and $D_{C}$.
} 
$B$; $A$ has a "very weak" record while $B$ has a "very strong" record. ${ }^{11}$ Therefore, individual $C$ should get a larger share of the credit on her project with $A$ than on her project with $B$ since $B$ is much stronger than $A$. Now, suppose we replaced $A$ and $B$ by an individual with their aggregate records and required $C$ 's credit to be unaffected. Then $C$ 's credit on both projects would be different despite these projects now being identical (involving the same teammates and having the same worth).

To state the axiom, we define the database resulting from the replacement of a group by an individual with the combined project record of the members of the group. For each database $D$ and each group of individuals $S \subseteq N(D)$ with the same collaborative projects, we denote by $D^{S}$ the database obtained by replacing all the members of $S$ by a single individual denoted by $i_{S}$ with the combined record of the members of $S$, all else equal. ${ }^{12}$

Aggregate strength: For each $S \subseteq N(D)$ such that each individual in $S$ has the same collaborative projects and each $i \in N(D) \backslash S, r_{i}\left(D^{S}\right)=r_{i}(D)$.

Thus, a rule satisfies aggregate strength if the allocation of credit for the individuals outside $S$ is identical after the members of $S$ have been replaced by individual $i_{S}$. An implication is that the credit allocated to $i_{S}$ for each project must be equal to the total credit that used to be allocated to the members of $S$. Aggregate strength is satisfied by the CoScore rule but not by the egalitarian rule. Aggregate strength is illustrated in Figure 5 for a database with three individuals; observe that the aggregated individuals, $A$ and $B$, have the same collaborative projects.

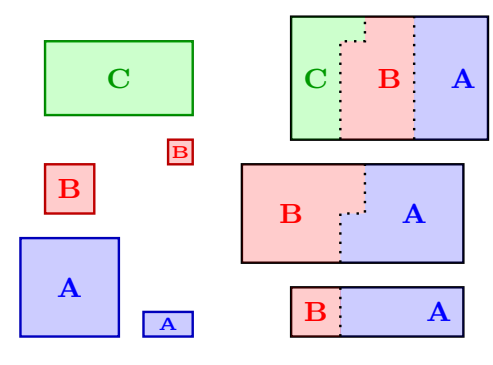

(i) Allocation of credit in the original database.

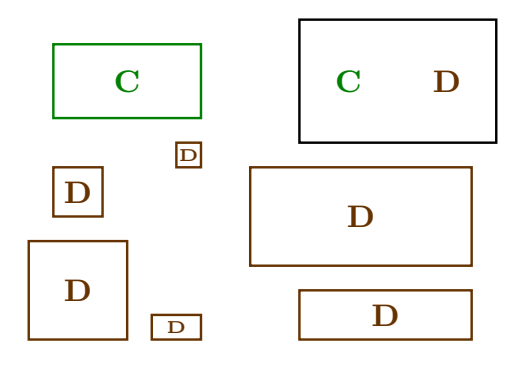

(ii) Reduced database after Ann and Bob are "aggregated" into Dan.

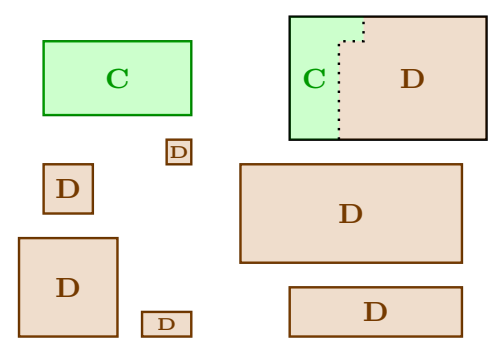

(iii) Aggregate Strength: Cat's credit on all her projects remains unaffected.

Figure 5. Aggregate Strength

\footnotetext{
${ }^{11}$ For example, beyond their collaborative project, their records could consist solely of individual projects, with each of $B$ 's projects having a greater worth than each of $A$ 's. Of course, the intuition applies more generally.

${ }^{12}$ Formally, $D^{S}$ comprises the same projects as $D, C\left(D^{S}\right)=C(D)$; for each project $p \in C\left(D^{S}\right)$, its worth is the same in the two databases, $w\left(p, D^{S}\right)=w(p, D)$ and, if no member of $S$ belongs to $T(p, D)$, its team is also the same $T\left(p, D^{S}\right)=T(p, D)$, otherwise $T\left(p, D^{S}\right)=[T(p, D) \backslash S] \cup\left\{i_{S}\right\}$ so the members of $S$ are replaced by individual $i_{S}$.
} 
We can now present the characterization of the CoScore rule.

Theorem 2. A rule satisfies consistency, aggregate worth, and aggregate strength if and only if it is the CoScore rule.

The properties in Theorem 2 are logically independent. See Appendix D.

Recall that the egalitarian rule also satisfies consistency and aggregate worth while the CoScore rule satisfies anonymity; these axioms are common to both rules. The difference between the rules thus boils down to that between own record only and aggregate strength.

\section{Extensions And CASE StUdies}

3.1. Extensions. CoScore can be extended to applications where individuals may not have solo projects and to incorporate additional observable information sharpening the assessment of individual productivity and credit. To illustrate, we introduce a family of scores extending CoScore that we will apply in our empirical case studies. This family is not meant to be the most general but simply to illustrate the potential enrichments of CoScore.

The additional observable information we consider are the ages of individuals and the time they participate in each project. For expositional ease, we henceforth drop the database index $D$, using the lighter notation of the Introduction. For each individual $i \in N$, we denote her age by $A_{i}$ and, for each project $p \in C$, we denote by $t_{i}(p)$ the amount of time she participated in the project. We assume that ages are strictly positive and that $t_{i}(p)>0$ if and only if $i$ belongs to $T(p)$. When the time individuals participate in a project cannot be observed, as in a coauthored scientific paper, we refer to participations as binary adopting the convention that, for each individual $i \in N$ and each project $p \in C, t_{i}(p)=1$ if $i$ belongs to $T(p)$ and $t_{i}(p)=0$ otherwise.

Each member of the family of scores defined next is parametrized by a scalar $\alpha \in[0,1]$; we call the score corresponding to parameter $\alpha$ the generalized $\alpha$-CoScore. It allocates a fixed share $\alpha$ of the credit on each project proportionally to the time participation of each teammate while the remaining share $(1-\alpha)$ is allocated proportionally to each teammate's endogenous score weighted by her time participation. The score is then defined as the average credit over the length of the individual's career. Formally, the generalized $\alpha$-CoScore defines the average productivity score of each individual by the system of equations

$$
s_{i}=\frac{1}{A_{i}} \sum_{p \in C_{i}}\left[\alpha \frac{t_{i}(p)}{\sum_{j \in T(p)} t_{j}(p)}+(1-\alpha) \frac{t_{i}(p) s_{i}}{\sum_{j \in T(p)} t_{j}(p) s_{j}}\right] w(p) \quad \forall i \in N
$$


The generalized $\alpha$-CoScore is well defined whenever $\alpha$ is strictly positive. Alternatively, if $\alpha$ equals zero, this can be ensured maintaining the assumption that each individual has at least one individual project with strictly positive worth. These are sufficient conditions for the system of equations (4) to have a unique solution (see Theorem 3 in Appendix A).

In fact, the well-definedness of CoScore depends critically on the presence of a positive constant term (that may be arbitrarily small) in the right-hand side of the equalities in its defining system of equations. For example, modifying (1) by adding any strictly positive term $\varepsilon_{i}$ as follows

$$
s_{i}=\varepsilon_{i}+\sum_{p \in C_{i}} \frac{s_{i}}{\sum_{j \in T(p)} s_{j}} w(p) \quad \forall i \in N
$$

is sufficient to guarantee the above system has a unique solution. Similarly, the first term inside the summation in (4) is a strictly positive constant when $\alpha>0$ and ensures welldefinedness. (See the proof of Theorem 3 in Appendix A.)

The formulation of the generalized $\alpha$-CoScore is flexible, including several interesting special cases:

(1) CoScore corresponds to the case where $\alpha=0$, all individuals have the same age normalized to one, and project participations are binary.

(2) The egalitarian score corresponds to the case where $\alpha=1$, all individuals have the same age normalized to one, and project participations are binary.

(3) When $\alpha=0$, the score allocates credit proportionally to the individual's expected contribution given her time participation $t_{i}(p) s_{i}$ since $s_{i}$ is interpreted as the individual's average productivity. We refer to this case as the generalized CoScore.

(4) When $\alpha=1$, credit is allocated proportionally to time participations. We refer to this case as the generalized egalitarian score. We use the term "egalitarian" since credit is allocated equally whenever all team members have the same time participations.

In practice, an adequate choice of $\alpha$ may reflect the structure of teamwork in an application. Low values of $\alpha$ may be appropriate where individual contributions determine team success. Credit should then be allocated mostly on the basis of individual quality. In contrast, when a team is only as strong as its weakest member, high $\alpha$ values and a correspondingly more egalitarian allocation may be appropriate. For example, success in football depends relatively more on an all round strong team than it does in basketball (as argued by Anderson and Sally, 2013) and this may call for a higher $\alpha$ in football. 
3.2. The productivity of academic researchers. Over the past thirty years, coauthorship has come to dominate the production of economic research (Laband and Tollison, 2000; Card and DellaVigna, 2013; Hamermesh, 2013), reflecting the general trend in science (Wuchty et al., 2007). A particular characteristic of economics is that authors are overwhelmingly listed alphabetically and that the specific role of each author on a paper is not specified. ${ }^{13}$ Not surprisingly, economists have expressed concerns on how to properly evaluate increasingly collaborative researchers (see, for example, Hamermesh, 2013; Liebowitz, 2013). This motivates the application of CoScore to the field of economics.

A project is now an academic paper, its members are the coauthors of the paper and its worth is the number of citations of the paper. For each paper, an author's participation is binary, with value 1 if she is an author on the paper and 0 otherwise. We use Thomson Reuters's Web of Science database to extract all published articles with at least one citation from 33 major journals in Economics over the period 1970 - 2015 (see Appendix E). The database includes 73732 articles, for a total of 29226 different authors. The share of coauthored papers increases steadily from only $20 \%$ in 1970 to almost $80 \%$ in 2015 , with the average number of coauthors going from 1.25 to 2.31. Papers with more than two authors, a rare occurence before the nineties, represent almost $40 \%$ of all papers published in 2015 . The increasing prominence of coauthorship also translates in the fraction of coauthored citations, which exhibits the same trend as the fraction of coauthored papers. Figure 6 shows the evolution of the fraction of coauthored papers and citations since 1970.
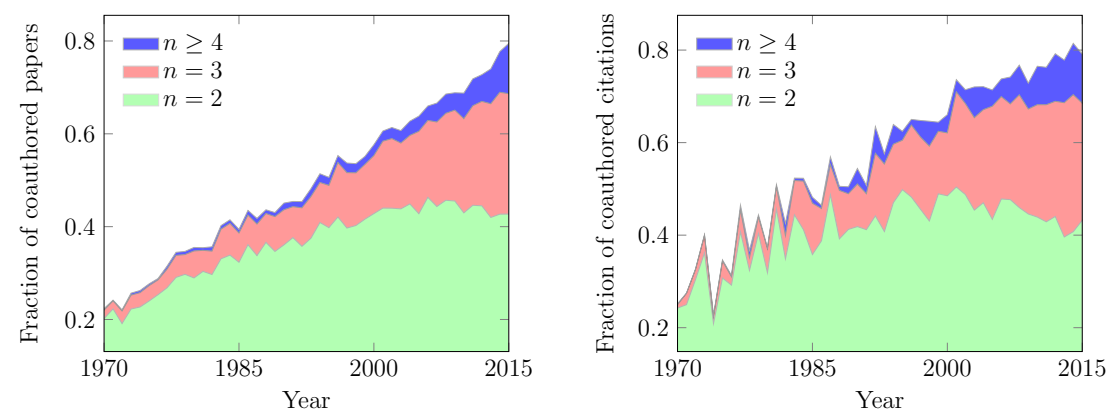

FiguRE 6. Fraction of coauthored papers (left) and citations (right) with 2, 3 and 4 or more authors from 1970 to 2015.

We compute the generalized $\alpha$-CoScore by repeatedly iterating the fixed point operator $\phi: x \in \mathbb{R}_{++}^{N} \mapsto \phi(x) \in \mathbb{R}_{++}^{N}$ defined by

\footnotetext{
${ }^{13}$ Engers et al. (1999) show how alphabetical name ordering, as opposed to relative contribution ordering, might emerge at equilibrium when coauthors bargain over the ordering after observing their relative contributions. In a recent paper Ray and Robson (2018) argue in favor of what they call certified randomization, where coauthors are either listed in order of contribution, or in random order. In the latter case, the randomization is made explicit by using a specific symbol between the name of the authors.
} 


$$
\phi_{i}(x)=\frac{1}{A_{i}} \sum_{p \in C_{i}}\left[\alpha \frac{1}{\# T(p)}+(1-\alpha) \frac{x_{i}}{\sum_{j \in T(p)} x_{j}}\right] w(p) \quad \forall i \in N
$$

starting from a vector of equal scores. The process converges quickly to the desired fixed point. We also compute the generalized egalitarian score (the generalized 1-CoScore), where the full credit on each paper is allocated equally between coauthors.

We observe a sharp divide between the generalized $\alpha$-CoScore (for $\alpha<1$ ) and the generalized egalitarian score. Figure 7 shows the cumulative distributions for the differential in ranks and scores between the generalized $\alpha$-CoScore and the generalized egalitarian score for $\alpha=0.1,0.3$, and 0.5. Accounting for the strength of coauthors in a given paper, not only their number, yields significant differences both in rankings and scores. If we focus on the 12557 authors who have at least one citation per year on average (all the authors who have an egalitarian score of at least one), individual ranks differ on average from the egalitarian ranks by $798.5,1342.6$ and 2345.6 positions, respectively, while individual scores differ on average by respectively $16.4 \%, 25.3 \%$ and $37.3 \%$. Interestingly, we observe that only a limited number of authors $(15.4 \%, 15.9 \%$ and $17.1 \%$, respectively) increase their score compared to the egalitarian benchmark.
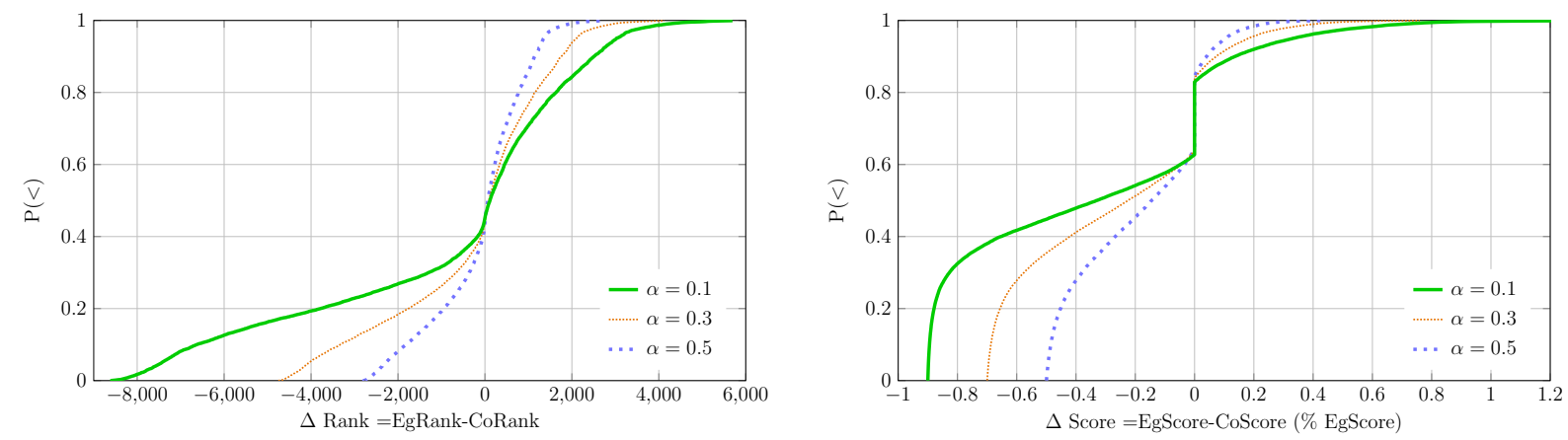

FiguRE 7. Distribution of the rank and score differentials between the generalized egalitarian score and the generalized $\alpha$-CoScore. Differences in scores are expressed in percentage of the generalized egalitarian score.

In order to understand the determinants of the generalized $\alpha$-CoScore, we run OLS regressions of the individual's total share of credit on coauthored papers (as allocated by the generalized $\alpha$-CoScore) on:

(i) the individual's average number of coauthors on joint papers (\# coauthors/paper),

(ii) the individual's total number of different coauthors (\# coauthors),

(iii) the individual's share of solo citations, and 
(iv) the log of the ratio between the individual's generalized egalitarian score $\left(E g a l_{i}\right)$ and the average generalized egalitarian score of her coauthors $\left(\operatorname{Egal}_{N_{i}}\right)$.

The ratio $E g a l_{i} / E g a l_{N_{i}}$ provides a first approximation of the individual's strength relative to her coauthors. ${ }^{14}$

TABLE 1. Regression analysis: allocated share of credit for the generalized $\alpha$-CoScore

\begin{tabular}{|c|c|c|c|c|c|}
\hline & (1) & $(2)$ & $(3)$ & (4) & $(5)$ \\
\hline & 0.1-CoScore & 0.3-CoScore & 0.5-CoScore & 0.7-CoScore & 0.9-CoScore \\
\hline \multirow[t]{2}{*}{ \# coauthors/paper } & $-0.0791^{* * *}$ & $-0.0871^{* * *}$ & $-0.0941^{* * *}$ & $-0.100^{* * *}$ & $-0.106^{* * *}$ \\
\hline & $(-37.86)$ & $(-66.41)$ & $(-104.67)$ & $(-147.23)$ & $(-174.17)$ \\
\hline \multirow[t]{2}{*}{ \# coauthors } & $0.00512^{* * *}$ & $0.00358^{* * *}$ & $0.00232^{* * *}$ & $0.00124^{* * *}$ & $0.000313^{* * *}$ \\
\hline & $(25.35)$ & $(28.25)$ & $(26.69)$ & (18.88) & $(5.31)$ \\
\hline \multirow[t]{2}{*}{ Solo share } & $0.153^{* * *}$ & $0.0968^{* * *}$ & $0.0597^{* * *}$ & $0.0330^{* * *}$ & $0.0134^{* * *}$ \\
\hline & $(30.56)$ & $(30.82)$ & $(27.73)$ & $(20.23)$ & $(9.15)$ \\
\hline \multirow[t]{2}{*}{$\log \left(\operatorname{Egal}_{i} / \operatorname{Egal}_{N_{i}}\right)$} & $0.150^{* * *}$ & $0.111^{* * *}$ & $0.0767^{* * *}$ & $0.0452^{* * *}$ & $0.0161^{* * *}$ \\
\hline & $(132.81)$ & $(156.69)$ & $(157.48)$ & $(122.29)$ & $(48.65)$ \\
\hline \multirow[t]{2}{*}{ Constant } & $0.604^{* * *}$ & $0.632^{* * *}$ & $0.656^{* * *}$ & $0.677^{* * *}$ & $0.695^{* * *}$ \\
\hline & $(108.09)$ & $(180.43)$ & $(273.18)$ & $(371.42)$ & $(426.53)$ \\
\hline Observations & 12134 & 12134 & 12134 & 12134 & 12134 \\
\hline Adjusted $R^{2}$ & 0.724 & 0.793 & 0.818 & 0.808 & 0.760 \\
\hline
\end{tabular}

$t$ statistics in parentheses

${ }^{*} p<0.05,{ }^{* *} p<0.01,{ }^{* * *} p<0.001$

The regression results indicate that lower values of $\alpha$ generally favor authors who have proved their individual quality by having published either a large fraction of single authored papers, with multiple groups of coauthors and/or with relatively weaker coauthors. However, as the following examples illustrate, authors might sometimes benefit from lower values of $\alpha$ even if some (or all!) of these conditions are not satisfied. For instance, author $A,{ }^{15}$ who has 74 papers for a total of 8113 citations, goes from being ranked $89^{\text {th }}$ with the generalized egalitarian score to being ranked $41^{\text {st }}$ with the generalized $\alpha$-CoScore (for $\alpha=0.1$ ), while her score is almost doubled, despite a very small fraction of single authored citations $(0.1 \%)$.

\footnotetext{
${ }^{14} \mathrm{~A}$ detailed decription of these variables is provided in the appendix.

${ }^{15}$ Authors $A, B$ and $C$ mentioned below are real authors taken from the database.
} 
In this case, author $A$ benefits from having worked with a large number of coauthors (66 coauthors), thus showing her ability to produce high quality work independently of the strength of a given coauthor. Author B, who has written 73 papers for a total of 7035 citations together with 27 different coauthors and has more than $50 \%$ of single authored citations, satisfies all the conditions mentioned before. Yet, her generalized $\alpha$-CoScore (for $\alpha=0.5,0.3$ and 0.1 ) is inferior to her generalized egalitarian score, and her ranking drops from $59^{\text {th }}$ to respectively $82^{\text {th }}, 90^{\text {th }}$ and $97^{\text {th }}$ because some of her most cited papers are coauthored with author $C$, a Nobel prize winner and ranked respectively $3^{\text {rd }}, 4^{\text {th }}$ and $4^{\text {th }}$ according to the $\alpha$-CoScore. Although having a large number of coauthors or a strong individual record is usually beneficial, it does not necessarily lead to an improved score over the generalized egalitarian score. What matters ultimately is the strength of a researcher's coauthors. Figure 8 illustrates the coauthorship network of author $C$.

3.2.1. Beyond economics. In economics or mathematics authors are listed alphabetically. However, in many other fields, the authorship order reflects either the importance or the nature of their contribution to the paper. In the biological sciences, for example, most recognition typically goes to the first and last authors, while the remaining authors' contributions can be harder to determine. In other fields like marketing, the convention is not clear (Maciejovsky et al., 2009). Increasingly, journals require the capacity of each coauthor (research design, research, data analysis, writing, etc) to be specified (Allen et al., 2014). Although conventions vary widely, even across sub-fields, this information is indicative of the contribution of each author and should play a role in the allocation of credit.

Our analysis can naturally be extended to account for ordered authorship and the capacity of each coauthor. A first alternative is allocating relatively more credit to authors who have a higher rank or capacity, by introducing paper-specific weights in the right hand side of the CoScore formula. A second alternative is separating the allocation of credit across groups of authors who have contributed to the paper in similar capacities. ${ }^{16}$ Each paper is subdivided into several artificial papers, one for each group, where the number of citations assigned to each paper (or group) depends on the relative importance of the associated capacity. ${ }^{17}$ CoScore and its extensions can then be applied to the resulting enlarged database.

\footnotetext{
${ }^{16}$ It is common for a paper to state that several of its authors contributed in the same capacities and for its authors not to be ranked strictly, for example, having two or more lead authors. A paper's authors can thus be ordered into contribution classes, whereby authors in the highest class are considered the most significant contributors, authors in the second highest class are the second most significant, and so forth.

${ }^{17}$ The credit allocation across groups of authors could follow the proposals of Stallings et al. (2013) or Hagen (2008).
} 


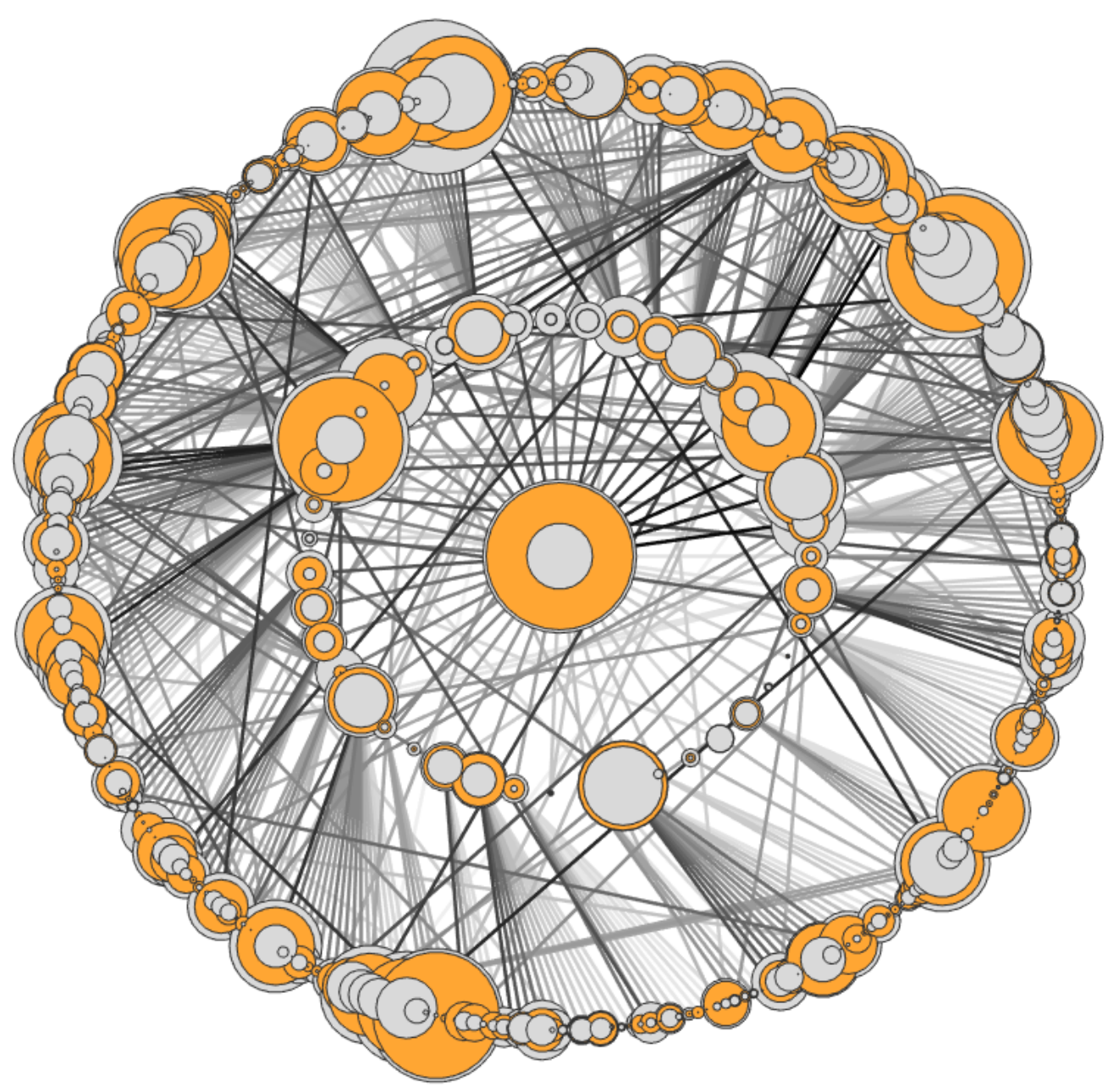

FiguRE 8. Coauthorship network of author $C$. Lines represent collaborations between pairs of authors, darker lines reflecting a higher number of joint citations. Nodes represent authors whose degree of separation from author $C$ (at the center) is at most 2. For each author (i) the size of the inner gray circle is proportional to the number of single-authored citations, (ii) the size of the outer gray circle is proportional to the total number of citations and (iii) the size of the orange ring is proportional to the number of jointly authored citations credited to the author by the generalized $\alpha$-CoScore (for $\alpha=0.1$ ).

3.2.2. Beyond citations as a measure of worth. Following a long tradition (Garfield, 1972), we measure the quality of a scientific paper by its total number of citations. Citations provide an objective metric that is based on observable data and can be used systematically on a large scale. ${ }^{18}$ However, citations have been shown to suffer from various shortcomings: (i) citation patterns and intensities differ substantially across fields and even sub-fields, making inter field comparisons problematic (Radicchi et al., 2008; Ellison, 2013), (ii) citations take time to accumulate, underestimating the value of recent papers (Wang et al., 2013), and (iii) citation counts treat each citation equally, ignoring that citations originating from more

\footnotetext{
${ }^{18}$ Perhaps the focus on citations is due to the lack of alternatives. Expert evaluations have been shown to suffer from systematic biases (Boudreau et al., 2016).
} 
influential papers reflect a higher value. In response, various alternative metrics have been proposed, respectively: (i) normalizing the citation count by the average per-paper citations in the field (sub-field) to insure comparability (Radicchi et al., 2008), (ii) measuring the quality of the journal where the paper was published using, for example, the impact factor or some other metric (Palacios-Huerta and Volij, 2014), (iii) discounting the citation count by the age of the paper, and (iv) giving more weight to citations originating from more influential papers, as measured by recursive network centrality measures (Pinski and Narin, 1976; Palacios-Huerta and Volij, 2004). CoScore and its extensions can be computed with any of these alternative measures of paper quality, providing complementary assessments of a scientist's productivity.

3.3. The productivity of sports players. We focus on the National Basketball League (NBA) because it consists of a closed pool of players and teams, which means it does not require comparing outcomes (wins) across leagues of possibly uneven value. ${ }^{19}$ We construct an exhaustive database consisting of the yearly records of all NBA teams from 1946 (the creation of the league) until 2011. A project now corresponds to a team for a given season (e.g. Boston Celtics in 2007/2008), its members are the players who played on the team that given season and its worth is the number of winning games in the season. ${ }^{20}$ For each project (team.season), a player's participation corresponds to the number of minutes spent on the court for the team during that season. The database consists of 1352 projects (team.season), for a total of 3800 different players.

We compute both the generalized egalitarian score and the generalized $\alpha$-CoScore for parameters $\alpha=0.1,0.3$, and 0.5 . As in the previous application, we observe significant differences both in terms of rankings and scores. Individual ranks (compared to the generalized egalitarian ranks) differ on average by respectively 72.5, 122.6 and 203.9 positions, while individual scores differ on average by respectively $32.6 \%, 51.3 \%$ and $79.4 \%$. Figure 9 shows the (cumulative) distributions for the differential in ranks and scores between the generalized $\alpha$-CoScore (for $\alpha=0.1,0.3$ and 0.5 ) and the generalized egalitarian score. As in the previous illustration, we observe that only a limited number of players increase their scores compared to the egalitarian benchmark $(14.2 \%, 13.1 \%$ and $11.5 \%$, respectively). These are the true most valuable players (MVPs) of the league, players who bring the most value to their teams and can lead them to victory even if they are not surrounded by other star players.

\footnotetext{
${ }^{19}$ In football, for example, players often participate in leagues of varying quality. The application of CoScore would then first require defining a scale so as to meaningfully compare wins across leagues.

${ }^{20}$ For simplicity, we do not account for playoff wins as this would require additional assumptions regarding their weight relative to wins in the regular season.
} 

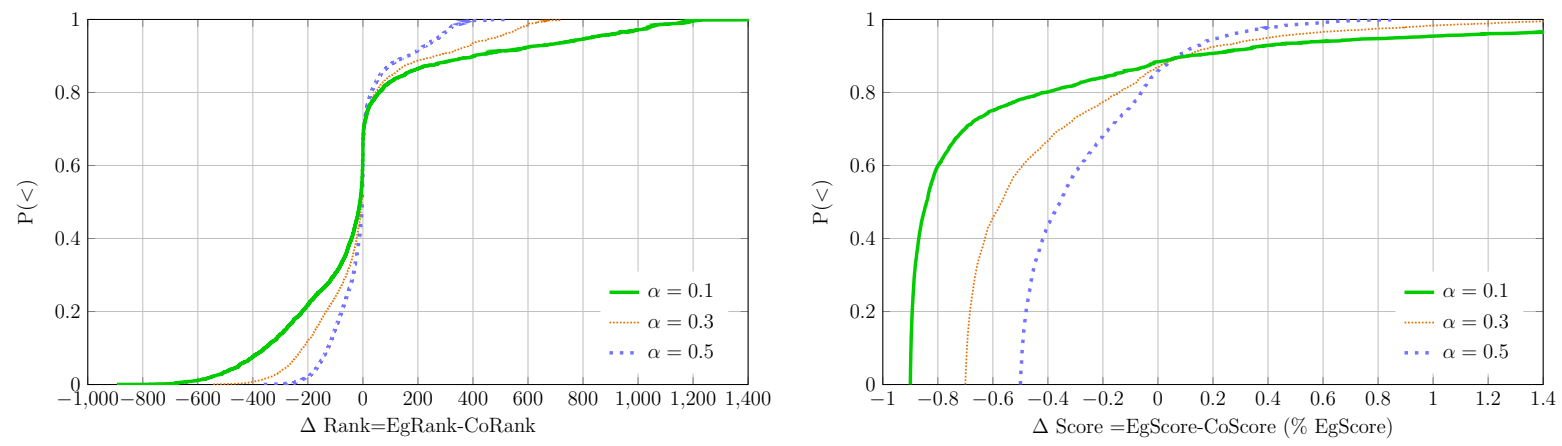

FiguRE 9. Distribution of the rank and score differentials between the generalized egalitarian score and generalized $\alpha$-CoScore. Differences in scores are expressed in percentage of the generalized egalitarian score.

We run OLS regressions of a player's share of credit under the generalized $\alpha$-CoScore on (i) the number of different teammates she has had (normalized by the length of her career) and (ii) the ratio between her generalized egalitarian score $\left(E_{g a l_{i}}\right)$ and the average generalized egalitarian score of her teammates $\left(\operatorname{Egal}_{N_{i}}\right){ }^{21}$

TABLE 2. Regression analysis: allocated share of credit

\begin{tabular}{lccccc}
\hline \hline & $(1)$ & $(2)$ & $(3)$ & $(4)$ & $(5)$ \\
& 0.1 -CoScore & 0.3 -CoScore & 0.5 -CoScore & 0.7 -CoScore & 0.9 -CoScore \\
\hline \# Teammates /Season & $0.00370^{* * *}$ & $0.00175^{* * *}$ & $0.000660^{* * *}$ & 0.0000243 & $-0.000362^{* * *}$ \\
& $(10.77)$ & $(11.02)$ & $(7.87)$ & $(0.41)$ & $(-6.44)$ \\
Egal $_{i} /$ Egal $_{N_{i}}$ & $0.160^{* * *}$ & $0.126^{* * *}$ & $0.106^{* * *}$ & $0.0932^{* * *}$ & $0.0844^{* * *}$ \\
& $(59.22)$ & $(101.23)$ & $(160.64)$ & $(201.75)$ & $(190.76)$ \\
& & & & \\
Constant & $-0.0873^{* * *}$ & $-0.0449^{* * *}$ & $-0.0199^{* * *}$ & $-0.00433^{* * *}$ & $0.00597^{* * *}$ \\
& $(-17.00)$ & $(-18.93)$ & $(-15.87)$ & $(-4.94)$ & $(7.10)$ \\
\hline Observations & 3800 & 3800 & 3800 & 3800 & 3800 \\
Adjusted $R^{2}$ & 0.529 & 0.779 & 0.904 & 0.940 & 0.936 \\
\hline \hline
\end{tabular}

$t$ statistics in parentheses

${ }^{*} p<0.05,{ }^{* *} p<0.01,{ }^{* * *} p<0.001$

The regression results indicate that lower values of $\alpha$ generally favor players who have proved their individual quality by winning (i) with a large number of teammates, and (ii) with relatively weaker teammates. This can be illustrated by looking at the successful campaign of the Boston Celtics in the 2007/2008 season. We focus on the ranking of the generalized

\footnotetext{
${ }^{21}$ Since there are no non-collaborative projects in this application, the allocated share of credit is equal to $A_{i} s_{i} / \sum_{p \in C_{i}} w(p)$.
} 
$\alpha$-CoScore for $\alpha=0.3$. The five most active players on the roster that season were Paul Pierce, Ray Allen, Kevin Garnett (dubbed "the big three"), Rajon Rondo and Kendrick Perkins. Paul Pierce, Ray Allen, and Kevin Garnett improve their ranking significantly, going from $41^{\text {th }}$ to $25^{\text {th }}, 70^{\text {th }}$ to $45^{\text {th }}$ and $34^{\text {th }}$ to $14^{\text {th }}$, respectively. In contrast, Rajon Rondo and Kendrick Perkins drop significantly, from $36^{\text {th }}$ to $206^{\text {th }}$ and $608^{\text {th }}$ to $1042^{\text {th }}$, respectively. The loss is not surprising for Kendrick Perkins, who has roughly half of the other players' generalized egalitarian score, and mechanically loses some credit to his stronger teammates. The loss is more puzzling for Rajon Rondo, who has the second highest generalized egalitarian score on the team, almost as high as Kevin Garnett's. However, while Rondo has only played with the big three (up until 2011), the big three have all played and won games with other sometimes much weaker teammates. Rondo's added value to the big three's success is not enough to compensate his lack of success outside the team. As a result, Rondo loses some credit to the benefit of Pierce, Allen, and Garnett.

\section{Conclusion}

We proposed a new method to quantify individual productivity in the presence of teamwork, CoScore. Our approach is well suited to large scale databases, exploiting variations in team composition and output. It provides a systematic and objective way of measuring individual productivity based only on the success of an individual's teams, information that can be observed and quantified precisely.

As we have illustrated for academic research and sports, CoScore and its extensions are practical and can easily be implemented systematically in large databases. Furthermore, they can be tailored to account for additional information such as the age and the degree of participation of individuals in their teams. Finally, the inferred credit can be used as an input for other well known indices that currently do not account for teamwork. For example, in the case of academic authorship, we could compute the $h, i$-10, Euclidean (Perry and Reny, 2016) or step-wise (Chambers and Miller, 2014) indices using the credits inferred by CoScore.

In practice, applying CoScore and its extensions to a particular database requires two important considerations: choosing a measure of team output and selecting an appropriate extension of CoScore.

Firstly, our analysis takes as input a well defined numerical measure of team output, such as the profit generated by a management team, the number of citations of an academic paper, etc. Depending on the application, different numerical measures of team success can be used, affecting the resulting individual productivity scores and rankings. For example, if a project is a film, its team is its cast, and its success is measured by box office revenue, the actors that systematically star in blockbusters will be ranked highly; in contrast, if success 
is measured using an index of critic ratings, artistically accomplished actors will be ranked highly. An application of CoScore thus first requires specifying what individual productivity we seek to measure and then selecting the appropriate measure of team success. Our focus in this paper is on deriving individual productivity given an appropriate team output measure. A significant amount of research already exists on deriving cardinal values for heterogeneous objects such as research projects, the value of a win in sports (Hochbaum and Levin, 2006) or scientific papers (Palacios-Huerta and Volij, 2004; Palacios-Huerta and Volij, 2014); these measures can all be used as inputs for CoScore and its extensions.

Secondly, depending on characteristics of teamwork in an application, different extensions of CoScore may be necessary. For example, the generalized $\alpha$-CoScore productivities and credits depend on the parameter $\alpha$ specifying the fraction of credit that is allocated exogenously across team members. As illustrated in our two numerical applications, different values for $\alpha$ may lead to significant differences in both ranking and scores. It is therefore important to understand how "best" to choose such a parameter. In the context of academic coauthorship, a possible strategy consists in estimating the parameter $\alpha$ that best predicts professional success, as measured by tenure decisions or salaries. Ellison (2013) follows a similar approach, investigating which academic productivity index in a family of $h$ - like indices best matches the professional placement of academic economists. The choice of the parameter $\alpha$ may also reflect other considerations, both normative and positive. For example, it may be desirable to guarantee a minimum level of credit for each individual contributing to a given project, or to provide individual incentives for team formation.

\section{REFERENCES}

Aczél, J. (2006). Lectures on Functional Equations and their Applications. Dover.

Adams, W. J. and J. L. Yellen (1976). Commodity bundling and the burden of monopoly. The Quarterly Journal of Economics, 475-498.

Allen, L., J. Scott, A. Brand, M. Hlava, and M. Altman (2014). Publishing: Credit where crdit is due. Nature 508(7496), 312-313.

Altman, A. and M. Tennenholtz (2005). Ranking systems: the PageRank axioms. In Proceedings of the 6th ACM conference on Electronic commerce, pp. 1-8. ACM.

Anderson, C. and D. Sally (2013). The Numbers Game: Why Everything You Know about Soccer is Wrong. Penguin original: Sports. Penguin Books.

Bergantiños, G. and J. D. Moreno-Ternero (2015). The axiomatic approach to the problem of sharing the revenue from museum passes. Games and Economic Behavior 89, 78-92. 
Bergantiños, G. and J. D. Moreno-Ternero (2018, February). Sharing the revenues from broadcasting sport events. Working Papers 18.02, Universidad Pablo de Olavide, Department of Economics.

Bikard, M., F. Murray, and J. S. Gans (2015). Exploring trade-offs in the organization of scientific work: Collaboration and scientific reward. Management Science 61(7), 14731495.

Bonacich, P. (1972). Factoring and weighting approaches to status scores and clique identification. Journal of Mathematical Sociology 2(1), 113-120.

Boudreau, K. J., E. C. Guinan, K. R. Lakhani, and C. Riedl (2016). Looking across and looking beyond the knowledge frontier: Intellectual distance, novelty, and resource allocation in science. Management Science 62(10), 2765-2783.

Card, D. and S. DellaVigna (2013). Nine facts about top journals in economics. Journal of Economic Literature 51(1), 144-161.

Chambers, C. P. and A. D. Miller (2014). Scholarly influence. Journal of Economic Theory $151,571-583$.

Demange, G. (2014). A ranking method based on handicaps. Theoretical Economics 9, 915-942.

Dequiedt, V. and Y. Zenou (2017). Local and consistent centrality measures in parameterized networks. Mathematical Social Sciences 88, 28-36.

Egghe, L. (2006). Theory and practice of the g-index. Scientometrics 69(1), 131-152.

Ellison, G. (2013). How does the market use citation data? the hirsch index in economics. American Economic Journal: Applied Economics 5, 63-90.

Engers, M., J. S. Gans, S. Grant, and S. P. King (1999). First-author conditions. Journal of Political Economy 107(4), 859-883.

Gans, J. S. and F. Murray (2013). Credit history: The changing nature of scientific credit. Technical report, National Bureau of Economic Research.

Gans, J. S. and F. Murray (2014). Markets for scientific attribution. Technical report, National Bureau of Economic Research.

Garfield, E. (1972, May). Citation analysis as a tool in journal evaluation. Science 178(4060), 471-479.

Ginsburgh, V. and I. Zang (2003). The museum pass game and its value. Games and Economic Behavior 43(2), 322-325.

Guillen, P., D. Merrett, and R. Slonim (2014). A new solution for the moral hazard problem in team production. Management Science 61(7), 1514-1530.

Hagen, N. T. (2008). Harmonic allocation of authorship credit: Source-level correction of bibliometric bias assures accurate publication and citation analysis. PLoS One 3(12), 
e4021.

Hamermesh, D. (2013). Six decades of top economics publishing. Journal of Economic Literature 51, 162-172.

Hirsch, J. E. (2005). An index to quantify an individual's scientific research output. Proceedings of the National Academy of Sciences 102(46), 16569-16572.

Hochbaum, D. S. and A. Levin (2006). Methodologies and algorithms for group-rankings decision. Management Science 52(9), 1394-1408.

Holmstrom, B. (1982). Moral hazard in teams. The Bell Journal of Economics, 324-340.

Jackson, M. (2008). Social and economic networks. Princeton University Press.

Jones, B. F. (2009). The burden of knowledge and the "death of the renaissance man": Is innovation getting harder? The Review of Economic Studies 76(1), 283-317.

Keener, J. P. (1993). The Perron-Frobenius theorem and the ranking of football teams. SIAM Review 35(1), 80-93.

Laband, D. N. and R. D. Tollison (2000). Intellectual collaboration. Journal of Political economy 108(3), 632-662.

Lehmann, S., A. Jackson, and B. Lautrum (2006, May). Measures for measures. Nature 444 (7122), 1003-1004.

Liebowitz, S. (2013). Willful blindness: The inefficient reward structure in academic research. Economic Inquiry 52(4), 1267-1283.

Maciejovsky, B., D. V. Budescu, and D. Ariely (2009). Research note: The researcher as a consumer of scientific publications: How do name-ordering conventions affect inferences about contribution credits? Marketing Science 28(3), 589-598.

Marchant, T. (2009). Score-based bibliometric rankings of authors. Journal of the American Society for Information Science and Technology 60(6), 1132-1137.

McAfee, R. P. and J. McMillan (1991). Optimal contracts for teams. International Economic Review, 561-577.

McAfee, R. P., J. McMillan, and M. D. Whinston (1989). Multiproduct monopoly, commodity bundling, and correlation of values. The Quarterly Journal of Economics 104(2), 371-383.

Newman, M. E. J. (2010). Networks: An Introduction. Oxford University Press.

Page, L., S. Brin, R. Motawani, and T. Winograd (1998). The PageRank citation ranking: Bringing order to the web. Working Paper, Stanford University.

Palacios-Huerta, I. and O. Volij (2004). The measurement of intellectual influence. Econometrica 72(3), 963-977.

Palacios-Huerta, I. and O. Volij (2014). Axiomatic measures of intellectual influence. International Journal of Industrial Organization 34, 85-90. 
Perry, M. and P. J. Reny (2016). How to count citations if you must. The American Economic Review $106(9), 2722-2741$.

Pinski, G. and F. Narin (1976). Citation influence for journal aggregates of scientific publications: Theory, with application to the literature of physics. Information processing $\&$ management 12(5), 297-312.

Price, D. (1981, May). Multiple authorship. Science 212(4498), 986.

Radicchi, F., S. Fortunato, and C. Castellano (2008). Universality of citation distributions: Toward an objective measure of scientific impact. Proceedings of the National Academy of Science 105(45), 17268-17272.

Ray, D. and A. Robson (2018). Certified random: A new order for coauthorship. American Economic Review 108(2), 489-520.

Sauer, R. D. (1988). Estimates of the returns to quality and coauthorship in economic academia. Journal of Political Economy 96(4), 855-866.

Shen, H. and A. Barabási (2014). Collective credit allocation in science. Proceedings of the National Academy of Sciences 111(34), 12325-12330.

Singh, J. and L. Fleming (2010). Lone inventors as sources of breakthroughs: Myth or reality? Management Science 56(1), 41-56.

Stallings, J., E. Vance, J. Yang, M. Vannier, J. Liang, L. Pang, L. Dai, I. Ye, and G. Wang (2013). Determining scientific impact using a collaboration index. Proceedings of the National Academy of Science 111(24), 9680-9685.

Thomson, W. (2001). On the axiomatic method and its recent applications to game theory and resource allocation. Social Choice and Welfare 18(2), 327-386.

Thomson, W. (2011). Consistency and its converse: an introduction. Review of Economic Design 15(4), 257 - 291.

Thomson, W. (2016). Consistent allocation rules. Monograph Series of the Econometric Society. Cambridge University Press, Forthcoming.

Wang, D., C. Song, and A.-L. Barabási (2013). Quantifying long-term scientific impact. Science 342(6154), 127-132.

Wuchty, S., B. Jones, and B. Uzzi (2007, May). The increasing dominance of teams in the production of knowledge. Science 316(2), 1036-1039.

Young, P. (1994). Equity: In Theory and Practice. Princeton University Press.

\section{Appendix A. Well Defined scores}

We only need to show that the score in (4) is well defined since this implies the other results, by considering the case where $\alpha=0$, each individual $i$ has age $A_{i}=1$ and has a binary time participation in each project $p$, with $t_{i}(p)=1$ if $i \in T(p)$ and $t_{i}(p)=0$ otherwise. 
Theorem 3. The system of equations (4) has a unique solution if either (i) $\alpha>0$ or (ii) each individual has a strictly positive individual contribution.

Proof. If $\alpha=1$, then equation system (4) reduces to

$$
s_{i}=\frac{1}{A_{i}} \sum_{p \in C_{i}} \frac{t_{i}(p)}{\sum_{j \in T(p)} t_{j}(p)} w(p) \quad \forall i \in N .
$$

This system trivially has a unique solution. Thus, from here on, consider the case where $\alpha<1$. Let $\phi: \mathbb{R}_{++}^{N} \rightarrow \mathbb{R}_{++}^{N}$ denote the function defined by setting, for each $x \in \mathbb{R}_{++}^{N}$,

$$
\phi_{i}(x)=\frac{1}{A_{i}} \sum_{p \in C_{i}}\left[\alpha \frac{t_{i}(p)}{\sum_{j \in T(p)} t_{j}(p)}+(1-\alpha) \frac{t_{i}(p) x_{i}}{\sum_{j \in T(p)} t_{j}(p) x_{j}}\right] w(p) \quad \forall i \in N .
$$

We now show that $\phi$ has a unique fixed point. Let $W=\sum_{p \in C} w(p)$ and, for each $i \in N$, let $I_{i}$ denote the collection of $i$ 's individual projects, all $p \in C$ such that $T(p)=\{i\}$. For each $i \in N$, let

$$
V_{i}=\sum_{p \in C_{i} \backslash I_{i}} \frac{t_{i}(p)}{\sum_{j \in T(p)} t_{j}(p)} w(p) \quad \text { and } \quad W_{i}=\sum_{p \in I_{i}} w(p) .
$$

Under assumption (i), for each $i \in N$, either $W_{i}>0$ or $\alpha V_{i}>0$ or both. Under assumption (ii), for each $i \in N, W_{i}>0$. Thus, independently of which assumption we make, for each $i \in N, \alpha V_{i}+W_{i}>0$. Note that, for each $x \in \mathbb{R}_{++}^{N}$ and each $i \in N, A_{i} \phi_{i}(x) \geq \alpha V_{i}+W_{i}>0$ and $\sum_{i \in N} A_{i} \phi_{i}(x)=W$. Thus, letting

$$
K=\left\{x \in \mathbb{R}^{N} \mid \forall i \in N, \quad \alpha V_{i}+W_{i} \leq A_{i} x_{i} \leq W\right\}
$$

we can redefine $\phi$ as a function mapping $K$ into $K$. Note that $K$ is compact and convex. Furthermore, $\phi$ is continuous on $K$. Thus, by Brouwer's fixed point theorem, $\phi$ has at least one fixed point. We now prove that this fixed point is unique. Let $x^{*} \in K$ denote any one of such fixed points. Note that the coordinates of $x^{*}$ are strictly positive because $K \subseteq \mathbb{R}_{++}^{N}$. Thus, by (6), for each $i \in N, \frac{\phi_{i}\left(x^{*}\right)}{x_{i}^{*}}=1$. That is,

$$
\sum_{p \in C_{i}} w(p)\left[\alpha \frac{t_{i}(p)}{x_{i}^{*} \sum_{j \in T(p)} t_{j}(p)}+(1-\alpha) \frac{t_{i}(p)}{\sum_{j \in T(p)} t_{j}(p) x_{j}^{*}}\right]=A_{i} \quad \forall i \in N .
$$

Let $f: \mathbb{R}_{++}^{N} \rightarrow \mathbb{R}$ denote the function defined by setting, for each $x \in \mathbb{R}_{++}^{N}$, 


$$
f(x) \equiv-\sum_{j \in N} A_{j} x_{j}+\sum_{p \in C} w(p)\left[\alpha \sum_{j \in T(p)} \ln \left(x_{j}\right) \cdot \frac{t_{j}(p)}{\sum_{k \in T(p)} t_{k}(p)}+(1-\alpha) \ln \left(\sum_{j \in T(p)} t_{j}(p) x_{j}\right)\right] .
$$

We first show that $f$ is strictly concave. Let $g: \mathbb{R}_{++}^{N} \rightarrow \mathbb{R}$ be defined, for each $x \in \mathbb{R}_{++}^{N}$, by $g(x)=\sum_{j \in N}\left[\alpha V_{j}+W_{j}\right] \cdot \ln \left(x_{j}\right)$. The function $g$ is strictly concave since its Hessian is negative definite because, for each $i \in N, \alpha V_{i}+W_{i}>0$. Let $C o \subseteq C$ consist of all of the collaborative projects in $C$. Let $h: \mathbb{R}_{++}^{N} \rightarrow \mathbb{R}$ be defined by setting, for each $x \in \mathbb{R}_{++}^{N}$,

$$
h(x)=-\sum_{j \in N} A_{j} x_{j}+(1-\alpha) \sum_{p \in C o} w(p) \ln \left(\sum_{j \in T(p)} t_{j}(p) x_{j}\right) .
$$

As the sum and composition of concave and affine functions, $h$ is concave. Since the sum of a strictly concave function and a concave function is strictly concave, $f=g+h$ is strictly concave.

By the strict concavity of $f$ and the fact that $\mathbb{R}_{++}^{N}$ is open and convex, $x^{* *}$ is the unique maximizer of $f$ over $\mathbb{R}_{++}^{N}$ if and only if $\nabla f\left(x^{* *}\right)=0$. That is,

$$
0=\frac{\partial f}{\partial x_{i}}\left(x^{* *}\right)=-A_{i}+\sum_{p \in C_{i}} w(p)\left[\alpha \frac{t_{i}(p)}{x_{i}^{* *} \sum_{j \in T(p)} t_{j}(p)}+(1-\alpha) \frac{t_{i}(p)}{\sum_{j \in T(p)} t_{j}(p) x_{j}^{* *}}\right] \quad \forall i \in N
$$

Thus, by (7), $x^{*}=x^{* *}$. Recall that $x^{*}$ is an arbitrary fixed point of $\phi$. By uniqueness of $x^{* *}$, we conclude that the fixed point of $\phi$ is indeed unique.

\section{Appendix B. Proof of Theorem 1}

Proof of Theorem 1. Let $r$ denote a rule satisfying anonymity and own record only. Let $\check{r}$ denote the egalitarian rule. Since it is obvious that $\check{r}$ satisfies the above axioms, it suffices to prove that $r=\check{r}$. For any rule and any database, only the individual involved in a noncollaborative project receives credit for it. Thus, the allocation of credit for non-collaborative projects coincides across rules. Thus, it suffices to show that the allocation of credit of $r$ and $\check{r}$ coincides across collaborative projects.

Step 1: Let $D$ denote a database such that $i, j \in N(D)$ and

$$
C_{i}(D) \backslash C(\{i\}, D) \underset{30}{=} C_{j}(D) \backslash C(\{j\}, D) .
$$


Let $D^{\prime}$ denote a database such that $N(D)=N\left(D^{\prime}\right)$ and

$C(D) \backslash[C(\{i\}, D) \cup C(\{j\}, D)]$ and $C\left(D^{\prime}\right) \backslash\left[C\left(\{i\}, D^{\prime}\right) \cup C\left(\{j\}, D^{\prime}\right)\right]$ coincide.

Then, for each $p \in C_{i}(D) \backslash C(\{i\}, D), r(p, D)=r\left(p, D^{\prime}\right)$.

Let $D$ denote a database where there are $i, j \in N(D)$ sharing the same set of collaborative projects, $C_{i}(D) \backslash C(\{i\}, D)=C_{j}(D) \backslash C(\{j\}, D)$ and let $p \in C_{i}(D) \backslash C(\{i\}, D)$. Let $D^{\prime}$ be any database such that $C\left(D^{\prime}\right) \backslash C\left(\{j\}, D^{\prime}\right)$ and $C(D) \backslash C(\{j\}, D)$ coincide. Since, for each $k \in N(D) \backslash\{j\}, C_{k}(D)$ and $C_{k}\left(D^{\prime}\right)$ coincide, by own record only, $r_{k}(p, D)=r_{k}\left(p, D^{\prime}\right)$. Thus, since the sum of the credits assigned by $r$ to the individuals involved in $p$ adds up to $w(p, D)=w\left(p, D^{\prime}\right), r_{j}(p, D)=r_{j}\left(p, D^{\prime}\right)$.

Let $D^{\prime \prime}$ be any database such that $C\left(D^{\prime \prime}\right) \backslash C\left(\{i\}, D^{\prime \prime}\right)$ and $C\left(D^{\prime}\right) \backslash C\left(\{i\}, D^{\prime}\right)$ coincide. Since, for each $k \in N(D) \backslash\{i\}, C_{k}\left(D^{\prime}\right)$ and $C_{k}\left(D^{\prime \prime}\right)$ coincide, by own record only, $r_{k}\left(p, D^{\prime}\right)=$ $r_{k}\left(p, D^{\prime \prime}\right)$. Thus, since the sum of the credits assigned by $r$ to the individuals involved in $p$ adds up to $w\left(p, D^{\prime}\right)=w\left(p, D^{\prime \prime}\right), r_{i}\left(p, D^{\prime}\right)=r_{i}\left(p, D^{\prime \prime}\right)$.

Altogether, $r(p, D)=r\left(p, D^{\prime \prime}\right)$. Since

$C(D) \backslash[C(\{i\}, D) \cup C(\{j\}, D)]$ and $C\left(D^{\prime \prime}\right) \backslash\left[C\left(\{i\}, D^{\prime \prime}\right) \cup C\left(\{j\}, D^{\prime \prime}\right)\right]$ coincide,

this concludes the proof of the Step 1.

Step 2: For each database D, each pair $i, j \in N(D)$ such that

$$
C_{i}(D) \backslash C(\{i\}, D)=C_{j}(D) \backslash C(\{j\}, D),
$$

and each $p \in C_{i}(D) \backslash C(\{i\}, D), r_{i}(p, D)=r_{j}(p, D)$.

Let $D$ denote a database where there are $i, j \in N(D)$ sharing the same set of collaborative projects, $C_{i}(D) \backslash C(\{i\}, D)=C_{j}(D) \backslash C(\{j\}, D)$. Let $p \in C_{i}(D) \backslash C(\{i\}, D)$ and $x=r(p, D)$. Let $D^{\prime}$ denote a database such that

i. $C\left(D^{\prime}\right) \backslash\left[C\left(\{i\}, D^{\prime}\right) \cup C\left(\{j\}, D^{\prime}\right)\right]$ and $C(D) \backslash[C(\{i\}, D) \cup C(\{j\}, D)]$ coincide,

ii. $C\left(\{i\}, D^{\prime}\right)$ and $C(\{j\}, D)$ coincide,

iii. $C\left(\{j\}, D^{\prime}\right)$ and $C(\{i\}, D)$ coincide.

Let $y=r\left(p, D^{\prime}\right)$. By Step 1, $y=x$. By anonymity, $y_{i}=x_{j}$ and $y_{j}=x_{i}$. Thus, $x_{i}=x_{j}$. Thus, $r_{i}(p, D)=r_{j}(p, D)$.

Step 3: For each database $D$, each $i \in N(D)$, and each $p \in C_{i}(D), r_{i}(p, D)=\frac{w(p, D)}{\# T(p, D)}$.

The proof is by induction.

Induction basis: For each $D$ and each $i \in N(D)$,

$$
\text { if } \# C_{i}(D) \backslash C(\{i\}, D)=1 \text {, then, for each } p \in C_{i}(D), r_{i}(p, D)=\frac{w(p, D)}{\# T(p, D)} \text {. }
$$


Let $D$ denote a database and $i \in N(D)$ be such that $C_{i}(D)$ contains a single collaborative project denoted by $p$. Let $D^{\prime}$ denote a database such that $N\left(D^{\prime}\right)=N(D)$ and such that

$$
C\left(D^{\prime}\right) \quad \text { and } \quad\{p\} \cup \bigcup_{j \in N(D)} C(\{j\}, D) \quad \text { coincide. }
$$

Thus, the only collaborative project in $D^{\prime}$ is $p$ and each individual has the same noncollaborative projects in $D$ as in $D^{\prime}$. By Step 2, for each pair $j, l \in T\left(p, D^{\prime}\right), r_{j}\left(p, D^{\prime}\right)=$ $r_{l}\left(p, D^{\prime}\right)$. Thus, $r_{i}\left(p, D^{\prime}\right)=\frac{w\left(p, D^{\prime}\right)}{\# T\left(p, D^{\prime}\right)}$. Since $C_{i}\left(D^{\prime}\right)$ and $C_{i}(D)$ coincide, by own record only,

$$
r_{i}(p, D)=r_{i}\left(p, D^{\prime}\right)=\frac{w\left(p, D^{\prime}\right)}{\# T\left(p, D^{\prime}\right)}=\frac{w(p, D)}{\# T(p, D)} .
$$

Induction hypothesis: Let $n$ denote a natural number. For each $D$ and each $i \in N(D)$,

$$
\text { if } \# C_{i}(D) \backslash C(\{i\}, D)<n \text {, then, for each } p \in C_{i}(D), r_{i}(p, D)=\frac{w(p, D)}{\# T(p, D)} \text {. }
$$

Let $D$ denote a database and $i \in N(D)$ be such that $\# C_{i}(D) \backslash C(\{i\}, D)=n$. We will show that, for each $p \in C_{i}(D) \backslash C(\{i\}, D), r_{i}(p, D)=\frac{w(p, D)}{\# T(p, D)}$.

Let $D^{\prime}$ denote a database such that $N\left(D^{\prime}\right)=N(D)$ and such that

$$
C\left(D^{\prime}\right) \text { and } \quad C_{i}(D) \backslash C(\{i\}, D) \cup \bigcup_{j \in N(D)} C(\{j\}, D) \text { coincide. }
$$

Thus, each collaborative project in $D^{\prime}$ involves $i$ and each individual has the same noncollaborative projects in $D$ as in $D^{\prime}$. Moreover, $C_{i}\left(D^{\prime}\right)$ and $C_{i}(D)$ coincide.

For each $j \in N(D)$, since $C_{j}\left(D^{\prime}\right) \backslash C\left(\{j\}, D^{\prime}\right) \subseteq C_{i}\left(D^{\prime}\right) \backslash C\left(\{i\}, D^{\prime}\right)$, we have $\# C_{j}\left(D^{\prime}\right) \backslash$ $C\left(\{j\}, D^{\prime}\right) \leq n$. Thus, for each $j \in N(D)$, there are two possible cases:

Case 1: $\# C_{j}\left(D^{\prime}\right) \backslash C\left(\{j\}, D^{\prime}\right)<n$. By the induction hypothesis,

$$
\text { for each } p \in C_{j}\left(D^{\prime}\right) \backslash C\left(\{j\}, D^{\prime}\right), r_{j}\left(p, D^{\prime}\right)=\frac{w\left(p, D^{\prime}\right)}{\# T\left(p, D^{\prime}\right)} \text {. }
$$

Case 2: $\# C_{j}\left(D^{\prime}\right) \backslash C\left(\{j\}, D^{\prime}\right)=n$. Then, $C_{j}\left(D^{\prime}\right) \backslash C\left(\{j\}, D^{\prime}\right)=C_{i}\left(D^{\prime}\right) \backslash C\left(\{i\}, D^{\prime}\right)$. Thus, by Step 2,

$$
\text { for each } p \in C_{j}\left(D^{\prime}\right) \backslash C\left(\{j\}, D^{\prime}\right), r_{j}\left(p, D^{\prime}\right)=r_{i}\left(p, D^{\prime}\right) \text {. }
$$

Let $p \in C_{i}\left(D^{\prime}\right) \backslash C\left(\{i\}, D^{\prime}\right)$ and $t=\# T\left(p, D^{\prime}\right)$. Let $t_{1}$ denote the number of $j \in T\left(p, D^{\prime}\right)$ such that $\# C_{j}\left(D^{\prime}\right) \backslash C\left(\{j\}, D^{\prime}\right)<n$ and $t_{2}$ denote the number of $j \in T\left(p, D^{\prime}\right)$ such that $\# C_{j}\left(D^{\prime}\right) \backslash C\left(\{j\}, D^{\prime}\right)=n$. Since, each collaborative project in $D^{\prime}$ involves $i$ and $i$ has exactly $n$ collaborative projects, $t=t_{1}+t_{2}$. By Case 1 , the rule awards $t_{1}$ individuals credit $\frac{w\left(p, D^{\prime}\right)}{t}$. By Case 2, the rule awards $t_{2}$ individuals credit $r_{i}\left(p, D^{\prime}\right)$. Since the total credit awarded adds up to the project's worth,

$$
t_{1} \cdot \frac{w\left(p, D^{\prime}\right)}{t}+t_{2} \cdot r_{i}\left(p, D^{\prime}\right)=w\left(p, D^{\prime}\right) .
$$


Thus, $r_{i}\left(p, D^{\prime}\right)=\frac{w\left(p, D^{\prime}\right)}{t}$. Since $C_{i}\left(D^{\prime}\right)$ and $C_{i}(D)$ coincide, by own record only,

$$
r_{i}(p, D)=r_{i}\left(p, D^{\prime}\right)=\frac{w\left(p, D^{\prime}\right)}{t}=\frac{w(p, D)}{\# T(p, D)}
$$

By induction, this establishes Step 3 and concludes the proof of the Theorem.

\section{Appendix C. Proof of Theorem 2}

We introduce two additional axioms implied by the axioms in Theorem 2. These axioms, stated independently, are useful in the arguments that follow. The first axiom requires credit on collaborative projects to be allocated proportionally to each individual's contribution when the database involves two individuals, each with one individual project and one collaborative project.

Proportionality: For each $D$ with $N(D)=\{i, j\}$ and $C(D)=\left\{p_{i}, p_{j}, p\right\}$ such that $T\left(p_{i}, D\right)=\{i\}, T\left(p_{j}, D\right)=\{j\}$, and $T(p, D)=\{i, j\}, \frac{r_{i}(p, D)}{r_{j}(p, D)}=\frac{w\left(p_{i}, D\right)}{w\left(p_{j}, D\right)}$.

The second axiom reflects the requirement that the name or indexing of projects is irrelevant to the credit assignment. Only the project worth and team-membership relations are taken into consideration.

Neutrality: For each pair $D, D^{\prime}$, if $N(D)=N\left(D^{\prime}\right)$ and $\sigma: C(D) \rightarrow C\left(D^{\prime}\right)$ is a bijection such that, for each $p \in C(D), w(p, D)=w\left(\sigma(p), D^{\prime}\right)$ and $T(p, D)=T\left(\sigma(p), D^{\prime}\right)$, then, for each $p \in C(D)$ and each $i \in N(D), r_{i}\left(\sigma(p), D^{\prime}\right)=r_{i}(p, D)$.

Remark 2. Aggregate worth implies neutrality. Aggregate strength implies anonymity.

Lemma 1. The CoScore rule satisfies consistency, aggregate worth, aggregate strength, and proportionality.

Proof. Let $\check{r}$ denote the CoScore rule and take a database $D$.

\section{Consistency}

Let $k \in N(D), x \equiv \check{r}(D)$, and $y \equiv \check{r}\left(D_{x}^{-k}\right)$. We need to prove that, for each $i \in N\left(D_{x}^{-k}\right)$ and each $p \in C\left(D_{x}^{-k}\right), x_{i}(p)=y_{i}(p)$. Using the definitions of $x$ and $y$ in Remark 1 , for each 
$p \in C\left(D_{x}^{-k}\right) \cap C_{k}(D)$ and each $i \in T\left(p, D_{x}^{-k}\right)$,

$$
\begin{aligned}
& y_{i}(p)=w\left(p, D_{x}^{-k}\right) \frac{\sum_{q \in C_{i}\left(D_{x}^{-k}\right)} y_{i}(q)}{\sum_{j \in T\left(p, D_{x}^{-k}\right)} \sum_{q \in C_{j}\left(D_{x}^{-k}\right)} y_{j}(q)} \\
& =\left[w(p, D)-x_{k}(p)\right] \frac{\sum_{q \in C_{i}(D)} y_{i}(q)}{\sum_{j \in T(p, D) \backslash\{k\}} \sum_{q \in C_{j}(D)} y_{j}(q)} \\
& =w(p, D)\left[1-\frac{\sum_{q \in C_{k}(D)} x_{k}(q)}{\sum_{j \in T(p, D)} \sum_{q \in C_{j}(D)} x_{j}(q)}\right] \frac{\sum_{q \in C_{i}(D)} y_{i}(q)}{\sum_{j \in T(p, D) \backslash\{k\}} \sum_{q \in C_{j}(D)} y_{j}(q)} \\
& =\left[w(p, D) \frac{\sum_{j \in T(p, D) \backslash\{k\}} \sum_{q \in C_{j}(D)} x_{j}(q)}{\sum_{j \in T(p, D)} \sum_{q \in C_{j}(D)} x_{j}(q)}\right] \frac{\sum_{q \in C_{i}(D)} y_{i}(q)}{\sum_{j \in T(p, D) \backslash\{k\}} \sum_{q \in C_{j}(D)} y_{j}(q)}
\end{aligned}
$$

and, for each $p \in C\left(D_{x}^{-k}\right) \backslash C_{k}(D)$ and each $i \in T\left(p, D_{x}^{-k}\right)$,

$$
y_{i}(p)=w\left(p, D_{x}^{-k}\right) \frac{\sum_{q \in C_{i}\left(D_{x}^{-k}\right)} y_{i}(q)}{\sum_{j \in T\left(p, D_{x}^{-k}\right)} \sum_{q \in C_{j}\left(D_{x}^{-k}\right)} y_{j}(q)}=w(p, D) \frac{\sum_{q \in C_{i}(D)} y_{i}(q)}{\sum_{j \in T(p, \mathcal{D})} \sum_{q \in C_{j}(D)} y_{j}(q)} .
$$

Using the definition of $x$ in Remark 1 , for each $p \in C\left(D_{x}^{-k}\right) \cap C_{k}(D)$ and each $i \in T\left(p, D_{x}^{-k}\right)$,

$$
\begin{aligned}
x_{i}(p) & =w(p, D)\left[\frac{\sum_{q \in C_{i}(D)} x_{i}(q)}{\sum_{j \in T(p, D)} \sum_{q \in C_{j}(D)} x_{j}(q)}\right] \\
& =\left[w(p, D) \frac{\sum_{j \in T(p, D) \backslash\{k\}} \sum_{q \in C_{j}(D)} x_{j}(q)}{\sum_{j \in T(p, D)} \sum_{q \in C_{j}(D)} x_{j}(q)}\right] \frac{\sum_{q \in C_{i}(D)} x_{i}(q)}{\sum_{j \in T(p, D) \backslash\{k\}} \sum_{q \in C_{j}(D)} x_{j}(q)}
\end{aligned}
$$

and, for each $p \in C\left(D_{x}^{-k}\right) \backslash C_{k}(D)$ and each $i \in T\left(p, D_{x}^{-k}\right)$,

$$
x_{i}(p)=w(p, D) \frac{\sum_{q \in C_{i}(D)} x_{i}(q)}{\sum_{j \in T(p, D)} \sum_{q \in C_{j}(D)} x_{j}(q)} .
$$

Let $z \in Z\left(D_{x}^{-k}\right)$ be such that, for each $p \in C\left(D_{x}^{-k}\right)$ and each $i \in N\left(D_{x}^{-k}\right), z_{i}(p)=x_{i}(p)$. Note that, by (10) and (11), $z$ satisfies the system of equations in (8) and (9). By Remark 1, (8) 
and (9) uniquely define $y$. Thus, $z=y$. Thus, for each $i \in N(D) \backslash\{k\}$ and each $p \in C\left(D_{x}^{-k}\right)$, $\check{r}_{i}\left(p, D_{x}^{-k}\right)=\check{r}_{i}(p, D)$. Thus, $\check{r}$ satisfies consistency.

\section{Aggregate worth}

Let $C \subseteq C(D)$ be such that for each pair $p, q \in C, T(p, D)=T(q, D)$ as in the hypothesis of aggregate worth. Let $S \subseteq N(D)$ be such that $C \subseteq C(S, D)$. Let $s=s(D)$ and $s^{\prime}=$ $s\left(D_{C}\right)$ denote the profiles of scores defined in equation system (3) for databases $D$ and $D_{C}$ respectively. Replacing all projects $C$ by a single project $p_{C}$ with total equal aggregate worth in equation system (3), we see that $s=s^{\prime}$. Thus, by the definition of $\check{r}$ as the CoScore rule, for each $i \in S$,

$$
\sum_{p \in C} \check{r}_{i}(p, D)=\sum_{p \in C} w(p, D) \frac{s_{i}}{\sum_{j \in T(p, D)} s_{j}}=w\left(p_{C}, D_{C}\right) \frac{s_{i}^{\prime}}{\sum_{j \in T\left(p, D_{C}\right)} s_{j}^{\prime}}=\check{r}_{i}\left(p_{C}, D_{C}\right)
$$

while for each $p \in C(D) \backslash C$ and each $i \in T(p, D)$,

$$
\check{r}_{i}(p, D)=w\left(p, D_{C}\right) \frac{s_{i}}{\sum_{j \in T(p, D)} s_{j}}=w\left(p, D_{C}\right) \frac{s_{i}^{\prime}}{\sum_{j \in T\left(p, D_{C}\right)} s_{j}^{\prime}}=\check{r}_{i}\left(p, D_{C}\right) .
$$

Thus, $\check{r}$ satisfies aggregate worth.

\section{Aggregate strength}

Let $S \subseteq N(D)$ be such that each individual in $S$ has the same list of collaborative projects: for each pair $i, j \in S, C_{i}(D) \backslash C(\{i\}, D)=C_{j}(D) \backslash C(\{j\}, D)$. By definition of the CoScore rule in Remark 1 , for each $p \in C(D)$ and each $i \in T(p, D)$,

$$
\check{r}_{i}(p, D)=w(p, D) \frac{\sum_{q \in C_{i}(D)} \check{r}_{i}(q, D)}{\sum_{j \in T(p, D)} \sum_{q \in C_{j}(D)} \check{r}_{j}(q, D)} .
$$

Let $z \in Z\left(D^{S}\right)$ be such that, for each $p \in C\left(D^{S}\right)$,

$$
z_{i_{S}}(p)=\sum_{i \in S} \check{r}_{i}(p, D) \quad \text { and, for each } j \in T(p, D) \backslash\left\{i_{S}\right\}, \quad z_{j}(p)=\check{r}_{j}(p, D) .
$$

Recall that $C\left(D^{S}\right)=C(D)$ and that, for each $p \in C(D), w\left(p, D^{S}\right)=w(p, D)$. Thus, by (12), for each $p \in C\left(D^{S}\right)$ and each $i \in T\left(p, D^{S}\right)$,

$$
z_{i}(p)=w\left(p, D^{S}\right) \frac{\sum_{q \in C_{i}\left(D^{S}\right)} z_{i}(q)}{\sum_{j \in T\left(p, D^{S}\right)} \sum_{q \in C_{j}\left(D^{S}\right)} z_{j}(q)}
$$


which means $z$ satisfies the system of equations defining the CoScore rule endogenously using (2) and Remark 1 for database $D^{S}$. By Remark $1, \check{r}\left(D^{S}\right)=z$. Thus, $\check{r}$ satisfies aggregate strength.

Proportionality

Suppose that $N(D)=\{i, j\}$ and that $C(D)=\left\{p_{i}, p_{j}, p\right\}$ are such that $T\left(p_{i}, D\right)=\{i\}$, $T\left(p_{j}, D\right)=\{j\}$, and $T(p, D)=\{i, j\}$. Then, by equation system (2) and Remark 1 ,

$$
\begin{aligned}
\check{r}_{i}(p, D) & =\frac{w\left(p_{i}, D\right)+\check{r}_{i}(p, D)}{w\left(p_{i}, D\right)+\check{r}_{i}(p, D)+w\left(p_{j}, D\right)+\check{r}_{j}(p, D)}, \\
\check{r}_{j}(p, D) & =\frac{w\left(p_{j}, D\right)+\check{r}_{j}(p, D)}{w\left(p_{i}, D\right)+\check{r}_{i}(p, D)+w\left(p_{j}, D\right)+\check{r}_{j}(p, D)}
\end{aligned}
$$

Dividing the first equation by the second one and rearranging yields the desired conclusion, $\check{r}_{i}(p, D) / \check{r}_{j}(p, D)=w\left(p_{i}, D\right) / w\left(p_{j}, D\right)$. Thus, $\check{r}$ satisfies proportionality.

Before proving the main theorem, we prove the following result:

Theorem 4. The only rule satisfying consistency, aggregate worth, and proportionality is the CoScore rule.

Proof. Let $r$ denote a rule satisfying consistency, aggregate worth, and proportionality. Then, by Remark 2, $r$ also satisfies neutrality. Let $\check{r}$ denote the CoScore rule. By Lemma 1, it suffices to prove that $r=\check{r}$.

Step 1: For each database $D$ such that $\# N(D)=2, r(D)=\check{r}(D)$.

Let database $D$ be such that $N(D)=\{i, j\}$ and let $K$ denote sum of the output of all collaborative projects in $D$; that is, $K=\sum_{p \in C(\{i, j\}, D)} w(p, D)$. Let $\mathbb{D}$ denote the family of databases such that each of its members involves the same authors as database $D$, the noncollaborative projects are the same as in $D$, and the sum of the output of the collaborative projects is also $K$ though the breakup of this aggregate worth into different projects may be different. Thus, $D^{\prime} \in \mathbb{D}$ if

- $N\left(D^{\prime}\right)=\{i, j\}$

- $C\left(\{i\}, D^{\prime}\right)=C(\{i\}, D)$ and $C\left(\{j\}, D^{\prime}\right)=C(\{j\}, D)$;

- for each $p \in C\left(\{i\}, D^{\prime}\right) \cup C\left(\{j\}, D^{\prime}\right), w\left(p, D^{\prime}\right)=w(p, D)$;

- $\sum_{p \in C\left(\{i, j\}, D^{\prime}\right)} w\left(p, D^{\prime}\right)=K$.

Note that $D \in \mathbb{D}$.

Step 1A: For each pair $D^{\prime}, D^{\prime \prime} \in \mathbb{D}$, each $p^{\prime} \in C\left(\{i, j\}, D^{\prime}\right)$ and each $p^{\prime \prime} \in C\left(\{i, j\}, D^{\prime \prime}\right)$, $w\left(p^{\prime}, D^{\prime}\right)=w\left(p^{\prime \prime}, D^{\prime \prime}\right)$ implies $r\left(p^{\prime}, D^{\prime}\right)=r\left(p^{\prime \prime}, D^{\prime \prime}\right)$. 
Let all of the notation be the same as in the statement of Step 1A. If $D^{\prime}$ contains a single collaborative project $p^{\prime}, K=w\left(p^{\prime}, D^{\prime}\right)=w\left(p^{\prime \prime}, D^{\prime \prime}\right)$. Thus, $D^{\prime \prime}$ also contains a single collaborative project $p^{\prime \prime}$ with worth $K$. By neutrality, $r\left(p^{\prime}, D^{\prime}\right)=r\left(p^{\prime \prime}, D^{\prime \prime}\right)$. We can repeat this argument if $D^{\prime \prime}$ contains a single collaborative project. Thus, from here on, suppose that each database contains at least two collaborative projects; that is, $C\left(\{i, j\}, D^{\prime}\right)$ and $C\left(\{i, j\}, D^{\prime \prime}\right)$ both have at least two elements.

Let $\hat{D} \in \mathbb{D}$ be such that $C(\{i, j\}, \hat{D})=\left\{p^{\prime}, \hat{p}\right\}, w\left(p^{\prime}, \hat{D}\right)=w\left(p^{\prime}, D^{\prime}\right)$, and $w(\hat{p}, \hat{D})=$ $K-w\left(p^{\prime}, D^{\prime}\right)$. By aggregate worth, replacing projects $C\left(\{i, j\}, D^{\prime}\right) \backslash\left\{p^{\prime}\right\}$ by $\hat{p}, r\left(p^{\prime}, D^{\prime}\right)=$ $r\left(p^{\prime}, \hat{D}\right)$. Similarly, let $\tilde{D} \in \mathbb{D}$ be such that $C(\{i, j\}, \tilde{D})=\left\{p^{\prime \prime}, \tilde{p}\right\}, w\left(p^{\prime \prime}, \tilde{D}\right)=w\left(p^{\prime \prime}, D^{\prime \prime}\right)$, and $w(\tilde{p}, \tilde{D})=K-w\left(p^{\prime \prime}, D^{\prime \prime}\right)$. By aggregate worth, replacing projects $C\left(\{i, j\}, D^{\prime \prime}\right) \backslash\left\{p^{\prime \prime}\right\}$ by $\tilde{p}, r\left(p^{\prime \prime}, D^{\prime \prime}\right)=r\left(p^{\prime \prime}, \tilde{D}\right)$. By neutrality, since the databases $\hat{D}$ and $\tilde{D}$ differ only in the indexing of projects,

$$
r\left(p^{\prime}, D^{\prime}\right)=r\left(p^{\prime}, \hat{D}\right)=r\left(p^{\prime \prime}, \tilde{D}\right)=r\left(p^{\prime \prime}, D^{\prime \prime}\right)
$$

Step 1B: For each pair $p, q \in C(D), \frac{r_{i}(p, D)}{r_{i}(q, D)}=\frac{w(p, D)}{w(q, D)}$.

Let $f:[0, K] \rightarrow \mathbb{R}$ be such that, for each $x \in[0, K]$,

$$
f(x)=r_{i}\left(p, D_{x}\right)
$$

where $D_{x} \in \mathbb{D}$ and $p \in C\left(\{i, j\}, D_{x}\right)$ is such that $w\left(p, D_{x}\right)=x$. By Step $1 \mathrm{~A}$, $f$ is well defined. By aggregate worth,

$$
f(x+y)=f(x)+f(y) \quad \forall x, y \in[0, K] \text { such that } x+y \leq K .
$$

Thus, $f$ satisfies the Cauchy functional equation and there is a constant $c_{K}$ such that, for each $x \in[0, K], f(x)=c_{K} x$ (see Theorem 3 on page 48 of Aczél, 2006). Thus, since $D \in \mathbb{D}$, for each pair $p, q \in C(D)$,

$$
r_{i}(p, D)=f(w(p, D))=c_{K} w(p, D) \text { and } r_{i}(q, D)=f(w(q, D))=c_{K} w(q, D) .
$$

This establishes Step 1B.

Step 1C: For each $p \in C(\{i, j\}, D), r_{i}(p, D)=\frac{w_{i}(D)}{w_{i}(D)+w_{j}(D)} w(q, D)$.

Let $D^{*}$ denote the database consisting of a collection of three projects, $C\left(D^{*}\right)=\left\{p_{i}, p_{i}, p_{i j}\right\}$, where $T\left(p_{i}, D^{*}\right)=\{i\}, T\left(p_{j}, D^{*}\right)=\{j\}$, and $T\left(p_{i j}, D^{*}\right)=\{i, j\}$. The output of each of these projects is given by

$$
w\left(p_{i}, D^{*}\right)=w_{i}(D), \quad w\left(p_{j}, D^{*}\right)=w_{j}(D), \quad w\left(p_{i j}, D^{*}\right)=K .
$$

Let $B \equiv C(\{i, j\}, D)$. 
By aggregate worth,

$$
r_{i}\left(p_{i j}, D^{*}\right)=\sum_{p \in B} r_{i}(p, D)
$$

By proportionality,

$$
r_{i}\left(p_{i j}, D^{*}\right)=\frac{w\left(p_{i}, D^{*}\right)}{w\left(p_{i}, D^{*}\right)+w\left(p_{j}, D^{*}\right)} w\left(p_{i j}, D^{*}\right) .
$$

By Step 1B, for each pair $p, q \in C(D)$,

$$
r_{i}(p, D)=\frac{w(p, D)}{w(q, D)} r_{i}(q, D)
$$

Thus, by (13),

$$
r_{i}\left(p_{i j}, D^{*}\right)=\sum_{p \in B} \frac{w(p, D)}{w(q, D)} r_{i}(q, D)=\frac{r_{i}(q, D)}{w(q, D)} \sum_{p \in B} w(p, D)=\frac{r_{i}(q, D)}{w(q, D)} w\left(p_{i j}, D^{*}\right) .
$$

Thus, by (14),

$$
\frac{w\left(p_{i}, D^{*}\right)}{w\left(p_{i}, D^{*}\right)+w\left(p_{j}, D^{*}\right)}=\frac{r_{i}(q, D)}{w(q, D)}
$$

Thus, for each $q \in B$,

$$
r_{i}(q, D)=\frac{w\left(p_{i}, D^{*}\right)}{w\left(p_{i}, D^{*}\right)+w\left(p_{j}, D^{*}\right)} w(q, D)=\frac{w_{i}(D)}{w_{i}(D)+w_{j}(D)} w(q, D)=\check{r}_{i}(q, D) .
$$

We conclude that $r(D)=\check{r}(D)$.

Step 2: For each database $D, r(D)=\check{r}(D)$.

Induction hypothesis: Let $n$ denote a strictly positive integer and suppose that, for each $D$ such that $\# N(D) \leq n, r(D)=\check{r}(D)$.

Let $D$ be such that $\# N(D)=n+1$ and $x \equiv r(D)$. For each $k \in N(D)$, let $N_{k} \equiv N(D) \backslash\{k\}$.

Let $k \in N(D)$. By consistency,

for each $p \in C(D) \backslash C(\{k\}, D)$ and each $i \in T(p, D) \backslash\{k\}, \quad r_{i}\left(p, D_{x}^{-k}\right)=x_{i}(p)$.

Thus, by the induction hypothesis, since $\# N_{k}=n$,

for each $p \in C(D) \backslash C(\{k\}, D)$ and each $i \in T(p, D) \backslash\{k\}, \quad x_{i}(p)=\check{r}_{i}\left(p, D_{x}^{-k}\right)$. 
Thus, for each $p \in C(D) \backslash C(\{k\}, D)$ and each $i \in T(p, D) \backslash\{k\}$,

$$
\begin{aligned}
x_{i}(p)=\check{r}_{i}\left(p, D_{x}^{-k}\right) & =w\left(p, D_{x}^{-k}\right) \frac{\sum_{q \in C_{i}\left(D_{x}^{-k}\right)} \check{r}_{i}\left(q, D_{x}^{-k}\right)}{\sum_{j \in T\left(p, D_{x}^{-k}\right)} \sum_{q \in C_{j}\left(D_{x}^{-k}\right)} \check{r}_{j}\left(q, D_{x}^{-k}\right)} \text { by definition of } \check{r}, \\
& =\left[w(p, D)-x_{k}(p)\right] \frac{\sum_{q \in C_{i}(D)} \check{r}_{i}\left(q, D_{x}^{-k}\right)}{\sum_{j \in T(p, D) \backslash\{k\}} \sum_{q \in C_{j}(D)} \check{r}_{j}\left(q, D_{x}^{-k}\right)} \text { by definition of } D_{x}^{-k}, \\
& =\left[w(p, D)-x_{k}(p)\right] \frac{\sum_{q \in C_{i}(D)}(q)}{\sum_{j \in T(p, D) \backslash\{k\}} \sum_{q \in C_{j}(D)} x_{j}(q)} \text { by }(15) .
\end{aligned}
$$

Thus, for each $p \in C(D) \backslash C(\{k\}, D)$ and each pair $i, j \in T(p, D) \backslash\{k\}$,

$$
\frac{x_{j}(p)}{x_{i}(p)}=\frac{\sum_{q \in C_{j}(D)} x_{j}(q)}{\sum_{q \in C_{i}(D)} x_{i}(q)} .
$$

Repeating the same argument for each $k \in N(D)$,

$$
\text { for each } p \in C(D) \text { and each pair } i, j \in T(p, D), \quad x_{j}(p)=x_{i}(p) \frac{\sum_{q \in C_{j}(D)} x_{j}(q)}{\sum_{q \in C_{i}(D)} x_{i}(q)} \text {. }
$$

Thus, for each $p \in C(D)$ and each $i \in T(p, D)$, summing over $j \in T(p, D)$,

$$
\sum_{j \in T(p, D)} x_{j}(p)=x_{i}(p) \frac{\sum_{j \in T(p, D)} \sum_{q \in C_{j}(D)} x_{j}(q)}{\sum_{q \in C_{i}(D)} x_{i}(q)} .
$$

Recall also that $x(p)$ is a credit allocation for project $p$ so $\sum_{j \in T(p, D)} x_{j}(p)=w(p, D)$. Thus, for each $p \in C(D)$ and each $i \in T(p, D)$,

$$
x_{i}(p)=w(p, D) \frac{\sum_{q \in C_{i}(D)} x_{i}(q)}{\sum_{j \in T(p, D)} \sum_{q \in C_{j}(D)} x_{j}(q)} .
$$

Hence, $x$ satisfies the system of equations (2) (using Remark 1) that endogenously defines the credit allocation recommended the CoScore rule for database $D$. However, by Remark 1 , 
$\check{r}(D)$ is the only solution to these conditions endogenously defining the CoScore rule for database $D$. Thus, $\check{r}(D)=x=r(D)$, as desired.

Lemma 2. If $r$ satisfies neutrality and aggregate strength, then it satisfies proportionality.

Proof. Suppose that $r$ satisfies neutrality and aggregate strength. By Remark 2, $r$ also satisfies anonymity. Let $\mathbb{D}$ denote the family of databases such that each of its databases has a collection of projects consisting of exactly one non-collaborative project for each individual and one collaborative project involving all of the individuals as team-members. Thus, for each $D \in \mathbb{D}$ and each $i \in N(D)$, there is a project denoted by $p_{i}$ involving only $i$ and a project denoted by $p$ involving all of $N(D)$.

Let $D \in \mathbb{D}$ be such that $N(D)=\{i, j\}, V \equiv w(p, D)$, and $W \equiv w\left(p_{i}, D\right)+w\left(p_{j}, D\right)$. For each $x \in[0, W]$, let $D_{x} \in \mathbb{D}$ denote a database such that

$$
w\left(p_{i}, D_{x}\right)=x, \quad w\left(p_{j}, D_{x}\right)=W-x, \quad w\left(p, D_{x}\right)=V .
$$

Let $a, b, c \in[0, W]$ be such that $a=b+c$. Let $D^{\prime} \in \mathbb{D}$ be such that $N\left(D^{\prime}\right)=\left\{i^{\prime}, i^{\prime \prime}, j\right\}$ where $i^{\prime}, i^{\prime \prime} \neq i$, and

$$
w\left(p_{i^{\prime}}, D^{\prime}\right)=b, \quad w\left(p_{i^{\prime \prime}}, D^{\prime}\right)=c, \quad w\left(p_{j}, D^{\prime}\right)=W-a, \quad w\left(p, D^{\prime}\right)=V .
$$

Since $a=b+c$, by aggregate strength,

$$
r_{i}\left(p, D_{a}\right)=r_{i^{\prime}}\left(p, D^{\prime}\right)+r_{i^{\prime \prime}}\left(p, D^{\prime}\right) .
$$

Let $D^{\prime \prime} \in \mathbb{D}$ be such that $N\left(D^{\prime \prime}\right)=\left\{i, j^{\prime}, j^{\prime \prime}\right\}$ where $j^{\prime}, j^{\prime \prime} \neq j$, and

$$
w\left(p_{i}, D^{\prime \prime}\right)=b, \quad w\left(p_{j^{\prime}}, D^{\prime \prime}\right)=c, \quad w\left(p_{j^{\prime \prime}}, D^{\prime \prime}\right)=W-a, \quad w\left(p, D^{\prime \prime}\right)=V .
$$

Since $c+(W-a)=W-b$, by aggregate strength, $r_{i}\left(p, D_{b}\right)=r_{i}\left(p, D^{\prime \prime}\right)$. By anonymity and neutrality, $r_{i}\left(p, D^{\prime \prime}\right)=r_{i^{\prime}}\left(p, D^{\prime}\right)$. Combining the two previous equations, $r_{i}\left(p, D_{b}\right)=$ $r_{i^{\prime}}\left(p, D^{\prime}\right)$. Similarly, $r_{i}\left(p, D_{c}\right)=r_{i^{\prime \prime}}\left(p, D^{\prime}\right)$. Thus, by (16),

$$
\text { if } a, b, c \in[0, W] \text { and } a=b+c \text {, then } \quad r_{i}\left(p, D_{a}\right)=r_{i}\left(p, D_{b}\right)+r_{i}\left(p, D_{c}\right) .
$$

Define $f:[0, W] \rightarrow[0, V]$ by setting, for each $x \in[0, W], f(x)=r_{i}\left(p, D_{x}\right)$. By (17), for each triple $a, b, c \in[0, W]$ such that $a=b+c, f(b+c)=f(b)+f(c)$. Thus, $f$ satisfies the Cauchy functional equation and there is a constant $\gamma$ such that, for each $x \in[0, W], f(x)=\gamma x$ (Theorem 3, page 48, Aczél, 2006). Since $D=D_{w\left(p_{i}, D\right)}, r_{i}(p, D)=\gamma w\left(p_{i}, D\right)$. By anonymity and neutrality, $r_{j}(p, D)=r_{j}\left(p, D_{w\left(p_{j}, D\right)}\right)=\gamma w\left(p_{j}, D\right)$. Thus,

$$
\frac{r_{i}(p, D)}{r_{j}(p, D)}=\frac{w\left(p_{i}, D\right)}{w\left(p_{j}, D\right)} .
$$

That is, $r$ satisfies proportionality.

Lemmas 1 and 2 and Theorem 4 enable a straightforward proof of Theorem 2. 
Proof of Theorem 2. By Lemma 1, the CoScore rule satisfies the properties in Theorem 2. Conversely, let $r$ denote a rule satisfying these properties. By Remark 2, $r$ satisfies neutrality. Thus, by Lemma 2, $r$ satisfies proportionality. Thus, by Theorem 4, $r$ is the CoScore rule.

\section{APPENDiX D. LOGICAL INDEPENDENCE}

We now introduce three rules to establish the logical independence of the axioms in Theorems 1 and 2:

i. The solo rule allocates the worth of each project proportionally to the total worth of an individual's solo projects: for each database $D$, each $p \in C(D)$, and each $i \in T(p, D)$,

$$
r_{i}(p, D)=\frac{\sum_{q \in C(\{i\}, D)} w(q, D)}{\sum_{j \in T(p, D)} \sum_{q \in C(\{j\}, D)} w(q, D)} w(p, D) .
$$

ii. The hybrid rule introduced next is a hybrid of the solo and CoScore rules allocating the worth of a predetermined project using the solo rule while applying the CoScore rule for every other project. Formally defining the rule requires some notation. Let $q$ denote a predetermined project and, for each database $D$, define the following associated databases:

$D_{-q}$ is such that $C\left(D_{-q}\right)=C(D) \backslash\{q\}$ and, for each $p \in C\left(D_{-q}\right), w\left(p, D_{-q}\right)=w(p, D)$ and $T\left(p, D_{-q}\right)=T(p, D)$. Thus, $D_{-q}$ denotes the database obtained from $D$ by removing project $q$.

$D_{+q}$ is such that $C\left(D_{+q}\right)=\{q\} \cup \bigcup_{i \in N(D)} C(\{i\}, D)$ and, for each $p \in C\left(D_{+q}\right)$, $w\left(p, D_{+q}\right)=w(p, D)$ and $T\left(p, D_{+q}\right)=T(p, D)$. Thus, $D_{+q}$ denotes the database obtained from $D$ by removing all collaborative projects except for $q$ if it is collaborative.

Let $\check{r}$ denote the CoScore rule and define the hybrid rule $r$ as follows: for each database $D$,

$$
r(q, D)=\check{r}\left(q, D_{+q}\right) \quad \text { and } \quad r(p, D)=\check{r}\left(p, D_{-q}\right) \quad \text { for each } p \in C(D) \backslash\{q\} .
$$

Note that if $q \notin C(D)$, the hybrid rule coincides with the CoScore rule.

iii. We now introduce a rule ensuring that the ratio between the credit of any two individuals involved in a project is constant. For each individual $i$ that may be involved in a database, let $c_{i}$ denote a strictly positive scalar and let $c$ denote the vector of such scalars specifying the corresponding scalar for each individual. We assume that there are $i$ and $j$ such that $c_{i} \neq c_{j}$, otherwise the rule will coincide with the egalitarian 
rule. For each such $c$, the fixed ratio rule (parametrized by $c$ ) is defined as follows: for each database $D$, each $p \in C(D)$, and each $i \in T(p, D)$,

$$
r_{i}(p, D)=\frac{c_{i}}{\sum_{j \in T(p, D)} c_{j}} w(p, D) .
$$

We emphasize that the parameters specifying a fixed ratio rule are exogenous.

The following table summarizes which axioms are satisfied by each of the rules discussed thus far.

\begin{tabular}{l|ccccc} 
& CoScore & Egalitarian & Solo & Hybrid & Fixed ratio \\
\hline anonymity & + & + & + & + & - \\
consistency & + & + & - & + & + \\
aggregate worth & + & + & + & - & + \\
aggregate strength & + & - & + & + & - \\
own record only & - & + & - & - & +
\end{tabular}

TABLE 3. An axiom in the row is satisfied by a rule in the column $(+)$ or it is not $(-)$.

Proposition 1. Consistency, aggregate worth, and aggregate strength are logically independent.

Proof. The egalitarian rule satisfies consistency and aggregate worth but not aggregate strength. The solo rule satisfies aggregate worth and aggregate strength but not consistency. While the hybrid rule satisfies consistency and aggregate strength, it does not aggregate worth since its predetermined project $q$ cannot be aggregated with other projects involving the same team without altering the credit allocation for the combined project and elsewhere in the database.

Proposition 2. Anonymity and own record only are logically independent.

Proof. The CoScore rule satisfies anonymity but not own record only. The fixed ratio rule satisfies own record only but not anonymity. This rule is not anonymous since the allocation of credit is determined by exogenous parameters $c_{i}$ that may be specific to each individual. It satisfies own record only since the allocation of credit in each project depends only on the team composition of the project, not on any other information in the database. 
The productivity of academic researchers. The data was retrieved from Thomson Reuters's Web of Science database on February 10th 2016. ${ }^{22}$ We extracted all published articles with at least one citation from the following 33 major journals in Economics over the period $1970-2015$ :

Econometrica, Quarterly Journal of Economics, Journal of Economic Literature, American Economic Review, Journal of Political Economy, Review of Economic Studies, Journal of Monetary Economics, Journal of Economic Theory, Games and Economic Behavior, Journal of Economics Perspectives, Journal of Econometrics, Rand Journal of Economics, Economic Theory, Journal of Labor Economics, Journal of Human Resources, Journal of Public Economics, Review of Economics and Statistics, Econometric Theory, Journal of Risk and Uncertainty, International Economic Review, Journal of Applied Econometrics, European Economic Review, International Journal of Game Theory, Social Choice and Welfare, Journal of Environmental Economics and Management, Economic Journal, Journal of International Economics, Journal of Economic Dynamics and Control, Journal of Mathematical Economics, Economic Inquiry, Scandinavian Journal of Economics, Economics Letters, Oxford Bulletin of Economics and Statistics.

The Web of Science database is not entirely consistent regarding the name of the authors. Authors are sometimes identified with their first initial only, all their initials and in some cases their full name. We choose here to retain the first initial only, which prevents the same author from being identified under two or more labels but means that authors with the exact same name and first initial are collapsed together. Note that the number of citations provided by Web of Science is usually smaller than the one of Google Scholar.

For each individual in $i$ the database, let $I_{i}$ denote her non-collaborative projects, all $p \in C$ such that $T(p)=\{i\}$ and, for each project $p$, let $r_{i}(p)$ denote her credit on project $p$ under the relevant generalized $\alpha$-CoScore. We can now define the variables used in the OLS regressions:

- i's share of credit on her collaborative projects,

$$
\frac{\sum_{p \in C_{i} \backslash I_{i}} r_{i}(p)}{\sum_{p \in C_{i} \backslash I_{i}} w(p)}
$$

\footnotetext{
${ }^{22} \mathrm{http}$ //ipscience.thomsonreuters.com/product/web-of-science/
} 
- $i$ 's average number of teammates in her collaborative projects,

$$
\frac{1}{\sum_{p \in C_{i}} w(p)} \cdot \sum_{p \in C_{i} \backslash I_{i}} \frac{w(p)}{\# T(p)}
$$

The average is computed by weighting the number of teammates on each paper by the paper's worth, then normalizing by the total worth of $i$ 's record.

- $i$ 's share of non-collaborative project worth,

$$
\frac{\sum_{p \in I_{i}} w(p)}{\sum_{p \in C_{i}} w(p)}
$$

- The average egalitarian score of $i$ 's teammates $\left(E g a l_{N_{i}}\right)$

$$
\text { Egal }_{N_{i}}=\frac{1}{\sum_{p \in C_{i} \backslash I_{i}} \frac{\# T(p)-1}{\# T(p)} w(p)} \cdot \sum_{p \in C_{i} \backslash I_{i}} w(p) \sum_{j \in T(p) \backslash\{i\}} E_{g a l_{j}}
$$

The average is computed by weighting each individual's egalitarian score by the total worth jointly produced with $i$. The first term is a normalizing factor. 